\title{
CONVERGENCE OF FUNCTIONALS AND ITS APPLICATIONS TO PARABOLIC EQUATIONS
}

\author{
GORO AKAGI
}

Received 11 December 2003

Asymptotic behavior of solutions of some parabolic equation associated with the $p$ Laplacian as $p \rightarrow+\infty$ is studied for the periodic problem as well as the initial-boundary value problem by pointing out the variational structure of the $p$-Laplacian, that is, $\partial \varphi_{p}(u)=-\Delta_{p} u$, where $\varphi_{p}: L^{2}(\Omega) \rightarrow[0,+\infty]$. To this end, the notion of Mosco convergence is employed and it is proved that $\varphi_{p}$ converges to the indicator function over some closed convex set on $L^{2}(\Omega)$ in the sense of Mosco as $p \rightarrow+\infty$; moreover, an abstract theory relative to Mosco convergence and evolution equations governed by time-dependent subdifferentials is developed until the periodic problem falls within its scope. Further application of this approach to the limiting problem of porous-medium-type equations, such as $u_{t}=\Delta|u|^{m-2} u$ as $m \rightarrow+\infty$, is also given.

\section{Introduction}

The so-called $p$-Laplacian $\Delta_{p}$ given below could be regarded as a nonlinear differential operator generalizing the usual linear Laplacian:

$$
\Delta_{p} u(x):=\nabla \cdot\left(|\nabla u(x)|^{p-2} \nabla u(x)\right), \quad 1<p<+\infty .
$$

This paper is motivated by the following naive question: what is the limit of $\Delta_{p}$ as $p \rightarrow$ $+\infty$ ? This limiting problem was studied by several authors and their results were applied in various fields; for example, growing sandpile model [2], macroscopic models for typeII superconductors $[1,4,13]$, and so on. In order to figure out the substantial features of this problem, we here recall the variational structure of $p$-Laplacian:

$$
-\Delta_{p} u=d I_{p}(u), \quad I_{p}(u):=\frac{1}{p} \int_{\Omega}|\nabla u(x)|^{p} d x \quad \forall u \in W_{0}^{1, p}(\Omega),
$$

where $d I_{p}(u)$ denotes the Fréchet derivative of the functional $I_{p}$ at $u$, and we intend to investigate the limit of the functional $I_{p}$ instead of $\Delta_{p}$ as $p \rightarrow+\infty$. 
However, it is easily expected that the limit of $I_{p}$ may not belong to the class of Fréchet differentiable functionals. On the other hand, from the viewpoint of studies on evolution equations, it is convenient for applications to extend $I_{p}$ on $L^{2}(\Omega)$ as follows:

$$
\varphi_{p}(u):= \begin{cases}I_{p}(u) & \text { if } u \in W_{0}^{1, p}(\Omega), \\ +\infty & \text { if } u \in L^{2}(\Omega) \backslash W_{0}^{1, p}(\Omega) .\end{cases}
$$

Then it is well known that $\varphi_{p}$ is no longer Fréchet differentiable on $L^{2}(\Omega)$, but lower semicontinuous and convex on $L^{2}(\Omega)$; moreover, its subdifferential $\partial_{L^{2}(\Omega)} \varphi_{p}(u)$ coincides with $-\Delta_{p} u$ in the distribution sense.

Several authors also studied the asymptotic behavior of solutions for the following initial-boundary value problem as $p \rightarrow+\infty$ :

$$
\begin{gathered}
\frac{\partial u}{\partial t}(x, t)-\Delta_{p} u(x, t)=0, \quad(x, t) \in \Omega \times(0, T), \\
u(x, t)=0, \quad(x, t) \in \partial \Omega \times(0, T), \\
u(x, 0)=u_{0}(x), \quad x \in \Omega .
\end{gathered}
$$

Here it is well known that (1.4) can be reduced to the following abstract Cauchy problem:

$$
\begin{aligned}
\frac{d u}{d t}(t)+\partial_{L^{2}(\Omega)} \varphi_{p}(u(t)) & =0 \quad \text { in } L^{2}(\Omega), 0<t<T, \\
u(0) & =u_{0} .
\end{aligned}
$$

According to the previous studies, for example, $[2,4]$, every solution $u_{p}$ of $(1.5)$ converges to $u$ as $p \rightarrow+\infty$ and the limit $u$ gives a solution of the following Cauchy problem:

$$
\begin{aligned}
\frac{d u}{d t}(t)+\partial_{L^{2}(\Omega)} \varphi_{\infty}(u(t)) & \ni 0 \text { in } L^{2}(\Omega), 0<t<T, \\
u(0) & =u_{0},
\end{aligned}
$$

where $\varphi_{\infty}$ is defined on $L^{2}(\Omega)$ by

$$
\varphi_{\infty}(u):= \begin{cases}0 & \text { if } u \in H_{0}^{1}(\Omega),|\nabla u|_{L^{\infty}(\Omega)} \leq 1, \\ +\infty & \text { otherwise. }\end{cases}
$$

Hence one can easily expect that $\varphi_{p}$ converges to $\varphi_{\infty}$ as $p \rightarrow+\infty$ in a certain sense; however, it is not so obvious in what sense it is realized. In this paper, we prove that $\varphi_{p}$ converges to $\varphi_{\infty}$ on $L^{2}(\Omega)$ in the sense of Mosco as $p \rightarrow+\infty$; moreover, we discuss the asymptotic behavior of solutions for (1.4) as $p \rightarrow+\infty$ in a more general setting. These results will be shown in Section 3.1 whereas the definition of Mosco convergence will be given in Section 2. Moreover, our method can also be applied to the porous medium equation

$$
\frac{\partial u}{\partial t}(x, t)-\Delta|u|^{m-2} u(x, t)=0, \quad(x, t) \in \Omega \times(0, T) .
$$

In Section 3.2, we deal with the asymptotic behavior of solutions for (1.8) as $m \rightarrow+\infty$. 
To formulate our results in an abstract form, we work in a more generalized setting, that is, we consider the following abstract evolution equations in a real Hilbert space $H$ governed by time-dependent subdifferential operators $\partial_{H} \varphi_{n}^{t}$ :

$$
\frac{d u_{n}}{d t}(t)+\partial_{H} \varphi_{n}^{t}\left(u_{n}(t)\right) \ni f_{n}(t) \quad \text { in } H, 0<t<T, n \in \mathbb{N}
$$

where $f_{n} \in L^{2}(0, T ; H), f_{n} \rightarrow f$ strongly in $L^{2}(0, T ; H)$, and $\varphi_{n}^{t}$ is a time-dependent proper lower semicontinuous convex functional from $H$ into $(-\infty,+\infty]$ such that $\varphi_{n}^{t} \rightarrow \varphi^{t}$ on $H$ in the sense of Mosco as $n \rightarrow+\infty$. For the case where $\varphi^{t}$ does not depend on $t$, that is, $\varphi^{t}=\varphi$, we can find related results in [3]. For the general case, we refer to Kenmochi [10].

However, all of the previous studies were done on the Cauchy problem for (1.9). As for the periodic problem for (1.9), there seems to be no attempt yet. The main objective here is to investigate the periodic problem as well as the Cauchy problem. The Cauchy problem has a unique solution, and the uniqueness of solution plays an essential role in deriving the convergence of $u_{n}$ as $n \rightarrow+\infty$ in $[3,10]$. On the other hand, in general, periodic solution is not unique. Hence the same procedure as in $[3,10]$ breaks down.

To cope with this difficulty, we introduce a remedy based on a compactness argument under a compactness assumption on the level set of $\left\{\varphi_{n}^{t}\right\}_{n \in \mathbb{N}}$. This result will be illustrated in the next section.

\section{Evolution equations and Mosco convergence}

This section deals with the following evolution equation $\left(\mathrm{E}\left(\varphi^{t}, f\right)\right)$ in a Hilbert space $H$. $\left(\mathrm{E}\left(\varphi^{t}, f\right)\right)$

$$
\frac{d u}{d t}(t)+\partial_{H} \varphi^{t}(u(t)) \ni f(t) \quad \text { in } H, 0<t<T,
$$

where $f \in L^{1}(0, T ; H)$ and $\partial_{H} \varphi^{t}$ is the subdifferential of a proper lower semicontinuous convex functional $\varphi^{t}: H \rightarrow(-\infty,+\infty]$ for every $t \in[0, T]$.

Throughout this paper, we denote by $\Psi(X)$ the set of all proper lower semicontinuous convex functionals $\phi$ from a Hilbert space $X$ into $(-\infty,+\infty]$, where "proper" means that $\phi \not \equiv+\infty$. Moreover, the subdifferential $\partial_{X} \phi$ of $\phi \in \Psi(X)$ is defined as follows:

$$
\partial_{X} \phi(u):=\left\{\xi \in X ; \phi(v)-\phi(u) \geq(\xi, v-u)_{X} \forall v \in D(\phi)\right\}
$$

where $(\cdot, \cdot)_{X}$ denotes the inner product of $X$ and $D(\phi)$ is the effective domain of $\phi$ given by

$$
D(\phi):=\{u \in X ; \phi(u)<+\infty\}
$$

Moreover, the domain $D\left(\partial_{X} \phi\right)$ of $\partial_{X} \phi$ is defined by

$$
D\left(\partial_{X} \phi\right):=\left\{u \in D(\phi) ; \partial_{X} \phi(u) \neq \varnothing\right\} .
$$


Now solutions of $\left(\mathrm{E}\left(\varphi^{t}, f\right)\right)$ are defined as follows.

Definition 2.1. A function $u \in C([0, T] ; H)$ is said to be a strong solution of $\left(\mathrm{E}\left(\varphi^{t}, f\right)\right)$ if the following are both satisfied:

(i) $u$ is an $H$-valued absolutely continuous function on $[0, T]$;

(ii) $u(t) \in D\left(\partial_{H} \varphi^{t}\right)$ for a.e. $t \in(0, T)$ and there exists a section $g(t) \in \partial_{H} \varphi^{t}(u(t))$ such that

$$
\frac{d u}{d t}(t)+g(t)=f(t) \quad \text { in } H \text { for a.e. } t \in(0, T)
$$

Moreover, a function $u \in C([0, T] ; H)$ is said to be a weak solution of $\left(\mathrm{E}\left(\varphi^{t}, f\right)\right)$ if there exist sequences $\left(f_{n}\right) \subset L^{1}(0, T ; H)$ and $\left(u_{n}\right) \subset C([0, T] ; H)$ such that $u_{n}$ is a strong solution of $\left(\mathrm{E}\left(\varphi^{t}, f_{n}\right)\right), f_{n} \rightarrow f$ strongly in $L^{1}(0, T ; H)$, and $u_{n} \rightarrow u$ strongly in $C([0, T] ; H)$.

We next introduce a notion of the convergence of functionals.

Definition 2.2. Let $X$ be a Hilbert space. Let $\left(\varphi_{n}\right)$ be a sequence in $\Psi(X)$ and let $\varphi \in \Psi(X)$. Then $\varphi_{n} \rightarrow \varphi$ on $X$ in the sense of Mosco as $n \rightarrow+\infty$ if the following conditions are all satisfied.

(1) For all $u \in D(\varphi)$, there exists a sequence $\left(u_{n}\right)$ in $X$ such that $u_{n} \rightarrow u$ strongly in $X$ and $\varphi_{n}\left(u_{n}\right) \rightarrow \varphi(u)$.

(2) Let $\left(u_{n}\right)$ be a sequence in $X$ such that $u_{n} \rightarrow u$ weakly in $X$. Then $\liminf _{n \rightarrow+\infty} \varphi_{n}\left(u_{n}\right) \geq$ $\varphi(u)$.

Remark 2.3. The second condition in Definition 2.2 is equivalent to the following.

$(2)^{\prime}$ Let $\left(u_{k}\right)$ be a sequence in $X$ such that $u_{k} \rightarrow u$ weakly in $X$ as $k \rightarrow+\infty$ and let $\left(n_{k}\right)$ be a subsequence of $(n)$. Then $\liminf _{k \rightarrow+\infty} \varphi_{n_{k}}\left(u_{k}\right) \geq \varphi(u)$.

Indeed, it is easily seen that (2) is derived immediately from $(2)^{\prime}$. Hence it suffices to show that (2) implies (2)'. Suppose that (2) holds but (2)' does not, that is, there exist a sequence $\left(u_{k}\right)$ and a subsequence $\left(n_{k}\right)$ of $(n)$ such that

$$
u_{k} \rightarrow u \quad \text { weakly in } X, \quad \liminf _{k \rightarrow+\infty} \varphi_{n_{k}}\left(u_{k}\right)<\varphi(u)
$$

Now define the sequence $\left(\tilde{u}_{n}\right)$ as follows: $\tilde{u}_{n}=u_{k}$ if $n \in\left[n_{k}, n_{k+1}\right)$ for each $k \in \mathbb{N}$. It then follows that $\tilde{u}_{n} \rightarrow u$ weakly in $X$ as $n \rightarrow+\infty$. Moreover, (2.6) yields

$$
\begin{array}{r}
\varphi(u)>\liminf _{k \rightarrow+\infty} \varphi_{n_{k}}\left(u_{k}\right)=\lim _{K \rightarrow+\infty} \inf _{k \geq K} \varphi_{n_{k}}\left(u_{k}\right) \\
\geq \lim _{K \rightarrow+\infty} \inf _{n \geq n_{K}} \varphi_{n}\left(\tilde{u}_{n}\right)=\liminf _{n \rightarrow+\infty} \varphi_{n}\left(\tilde{u}_{n}\right),
\end{array}
$$

which contradicts (2). Hence (2) implies (2)'. 
In the following two subsections, we discuss the existence and uniqueness of solutions $u_{n}$ for $\left(\mathrm{E}\left(\varphi_{n}^{t}, f_{n}\right)\right)$ and the convergence of $u_{n}$ as $n \rightarrow+\infty$ for the periodic problem as well as the Cauchy problem. To this end, we fix notations. From now on, we write $\left\{\varphi^{t}\right\}_{t \in[0, T]} \in$ $\Psi(\alpha, \beta)$ for some functions $\alpha, \beta:[0,+\infty) \times[0, T] \rightarrow \mathbb{R}$ if the following hold true:

(i) $\varphi^{t} \in \Psi(H)$ for all $t \in[0, T]$;

(ii) there exists $\delta>0$; for all $t_{0} \in[0, T]$ and all $u_{0} \in D\left(\varphi^{t_{0}}\right)$, there exists a function $u$ from $I_{\delta}\left(t_{0}\right):=\left[t_{0}-\delta, t_{0}+\delta\right] \cap[0, T]$ into $H$; for all $t \in I_{\delta}\left(t_{0}\right)$ and all $r \geq\left|u_{0}\right|_{H}$,

$$
\begin{gathered}
\left|u(t)-u_{0}\right|_{H} \leq\left|\alpha(r, t)-\alpha\left(r, t_{0}\right)\right|\left\{\left|\varphi^{t_{0}}\left(u_{0}\right)\right|+1\right\}^{1 / 2}, \\
\varphi^{t}(u(t)) \leq \varphi^{t_{0}}\left(u_{0}\right)+\left|\beta(r, t)-\beta\left(r, t_{0}\right)\right|\left\{\left|\varphi^{t_{0}}\left(u_{0}\right)\right|+1\right\} .
\end{gathered}
$$

Moreover, we say $\left\{\varphi^{t}\right\}_{t \in[0, T]} \in B\left(\alpha, \beta, C_{0},\left\{M_{r}\right\}_{r \geq 0}\right)$ for some functions $\alpha, \beta:[0,+\infty) \times$ $[0, T] \rightarrow \mathbb{R}$ and constants $C_{0},\left\{M_{r}\right\}_{r \geq 0}$ if the following are all satisfied.

(i) $\left\{\varphi^{t}\right\}_{t \in[0, T]} \in \Psi(\alpha, \beta)$.

(ii) $\varphi^{t}(u) \geq-C_{0}\left(|u|_{H}+1\right)$ for all $u \in H$ and all $t \in[0, T]$.

(iii) There exists a function $h:[0, T] \rightarrow H$ such that

$$
\sup _{t \in[0, T]}\left\{|h(t)|_{H}+\left|\varphi^{t}(h(t))\right|\right\}+\left(\int_{0}^{T}\left|\frac{d h}{d t}(t)\right|_{H}^{2} d t\right)^{1 / 2} \leq C_{0} .
$$

(iv) For every $r \in[0,+\infty)$, it follows that

$$
\int_{0}^{T}|\dot{\alpha}(r, t)|^{2} d t+\int_{0}^{T}|\dot{\beta}(r, t)| d t \leq M_{r}
$$

where $\dot{\alpha}$ and $\dot{\beta}$ denote $\partial \alpha / \partial t$ and $\partial \beta / \partial t$, respectively.

Now let $\left\{\varphi^{t}\right\}_{t \in[0, T]} \in \Psi(\alpha, \beta)$ be such that $\alpha(r, \cdot) \in W^{1,2}(0, T)$ and $\beta(r, \cdot) \in W^{1,1}(0, T)$ for all $r \in[0,+\infty)$ and introduce the following functional $\Phi^{S}$ defined on $\mathscr{H}_{S}:=L^{2}(0, S ; H)$ for any $S \in(0, T]$ :

$$
\Phi^{S}(u):= \begin{cases}\int_{0}^{S} \varphi^{t}(u(t)) d t & \text { if the function } t \longmapsto \varphi^{t}(u(t)) \in L^{1}(0, S) \\ +\infty & \text { otherwise. }\end{cases}
$$

Then we see that $\Phi^{S} \in \Psi\left(\mathscr{H}_{S}\right)$. Moreover, [9, Proposition 1.1] implies that for any $u, f \in$ $\mathscr{H}_{S}$,

$$
f \in \partial_{\mathscr{H}_{S}} \Phi^{S}(u) \Longleftrightarrow f(t) \in \partial_{H} \varphi^{t}(u(t)) \text { for a.e. } t \in(0, S) \text {. }
$$

The following proposition plays an important role in investigating the convergence of strong solutions $u_{n}$ for $\left(\mathrm{E}\left(\varphi_{n}^{t}, f_{n}\right)\right)$ as $n \rightarrow+\infty$. For its proof, we refer to [10, Proposition 2.7.1]. 
912 Convergence of functionals and parabolic equations

Proposition 2.4 [10]. For every $n \in \mathbb{N}$, let $\left\{\varphi_{n}^{t}\right\}_{t \in[0, T]} \in B\left(\alpha_{n}, \beta_{n}, C_{0},\left\{M_{r}\right\}_{r \geq 0}\right)$ and $\left\{\varphi^{t}\right\}_{t \in[0, T]} \in \Psi(\alpha, \beta)$ be such that $\alpha_{n}(r, \cdot), \alpha(r, \cdot) \in W^{1,2}(0, T)$ and $\beta_{n}(r, \cdot), \beta(r, \cdot) \in$ $W^{1,1}(0, T)$ for every $r \in[0,+\infty)$. Suppose that $\varphi_{n}^{t} \rightarrow \varphi^{t}$ on $H$ in the sense of Mosco for every $t \in[0, T]$ as $n \rightarrow+\infty$. Then for any $S \in(0, T]$, it follows that

(1) for each $u \in D\left(\Phi^{S}\right)$, there exists a sequence $\left(u_{n}\right)$ in $\mathscr{H}_{S}$ such that $u_{n} \rightarrow u$ strongly in $\mathcal{H}_{S}$ and $\Phi_{n}^{S}\left(u_{n}\right) \rightarrow \Phi^{S}(u)$, where $\Phi_{n}^{S}$ is defined by (2.11) with $\varphi^{t}$ replaced by $\varphi_{n}^{t}$;

(2) let $\left(u_{k}\right)$ be a sequence in $\mathcal{H}_{S}$ such that $\left(u_{k}\right)$ is bounded in $L^{\infty}(0, S ; H)$ and $u_{k}(t) \rightarrow u(t)$ weakly in $H$ for a.e. $t \in(0, S)$ as $k \rightarrow+\infty$ and let $\left(n_{k}\right)$ be a subsequence of $(n)$. Then $\liminf _{k \rightarrow+\infty} \Phi_{n_{k}}^{S}\left(u_{k}\right) \geq \Phi^{S}(u)$.

Throughout the present paper, we denote by $C$ or $C_{i}(i=1,2, \ldots)$ nonnegative constants which do not depend on the elements of the corresponding space or set.

2.1. Cauchy problem. In this subsection, we consider the following Cauchy problem $\left(\mathrm{CP}\left(\varphi^{t}, f, u_{0}\right)\right)$ in a Hilbert space $H$.

$\left(\mathrm{CP}\left(\varphi^{t}, f, u_{0}\right)\right)$

$$
\begin{gathered}
\frac{d u}{d t}(t)+\partial_{H} \varphi^{t}(u(t)) \ni f(t) \quad \text { in } H, 0<t<T, \\
u(0)=u_{0},
\end{gathered}
$$

where $\varphi^{t} \in \Psi(H)$ for all $t \in[0, T], f \in L^{1}(0, T ; H)$, and $u_{0} \in H$.

We first give a definition of solutions for $\left(\mathrm{CP}\left(\varphi^{t}, f, u_{0}\right)\right)$ as follows.

Definition 2.5. A function $u \in C([0, T] ; H)$ is said to be a strong (resp., weak) solution of $\left(\mathrm{CP}\left(\varphi^{t}, f, u_{0}\right)\right)$ if $u$ is a strong (resp., weak) solution of $\left(\mathrm{E}\left(\varphi^{t}, f\right)\right)$ such that $u(t) \rightarrow u_{0}$ strongly in $H$ as $t \rightarrow+0$.

As for the existence of solutions for $\left(\mathrm{CP}\left(\varphi^{t}, f, u_{0}\right)\right)$, we here employ the following.

Theorem 2.6 [10]. Let $\left\{\varphi^{t}\right\}_{t \in[0, T]} \in \Psi(\alpha, \beta)$ be such that $\alpha(r, \cdot) \in W^{1,2}(0, T)$ and $\beta(r, \cdot)$ $\in W^{1,1}(0, T)$ for every $r \in[0,+\infty)$. Then for all $f \in L^{1}(0, T ; H)$ and $u_{0} \in{\overline{D\left(\varphi^{0}\right)}}^{H}$, $\left(\mathrm{CP}\left(\varphi^{t}, f, u_{0}\right)\right)$ has a unique weak solution $u$ such that the function $t \mapsto \varphi^{t}(u(t))$ is integrable on $(0, T)$. In particular, if $f \in L^{2}(0, T ; H)$, then the weak solution $u$ satisfies

$$
\sqrt{t} \frac{d u}{d t} \in L^{2}(0, T ; H), \quad \sup _{t \in[0, T]} t \varphi^{t}(u(t))<+\infty .
$$

Moreover, if $f \in L^{2}(0, T ; H)$ and $u_{0} \in D\left(\varphi^{0}\right)$, then the unique weak solution $u$ becomes a strong solution of $\left(\mathrm{CP}\left(\varphi^{t}, f, u_{0}\right)\right)$ such that

$$
\frac{d u}{d t} \in L^{2}(0, T ; H), \quad \sup _{t \in[0, T]} \varphi^{t}(u(t))<+\infty .
$$

On account of Proposition 2.4, Kenmochi also proved the following result on the convergence of solutions $u_{n}$ for $\left(\mathrm{CP}\left(\varphi_{n}^{t}, f_{n}, u_{0, n}\right)\right)$ as $n \rightarrow+\infty$. Its proof can be found in [10, Theorem 2.7.1]. 
Theorem 2.7 [10]. Under the same assumptions as in Proposition 2.4, let $\left(f_{n}\right)$ and $\left(u_{0, n}\right)$ be sequences in $L^{2}(0, T ; H)$ and ${\overline{D\left(\varphi_{n}^{0}\right)}}^{H}$, respectively, such that $f_{n} \rightarrow f$ strongly in $L^{2}(0, T ; H)$ and $u_{0, n} \rightarrow u_{0} \in{\overline{D\left(\varphi^{0}\right)}}^{H}$ strongly in $H$. Then the unique weak solution $u_{n}$ of $\left(\operatorname{CP}\left(\varphi_{n}^{t}, f_{n}, u_{0, n}\right)\right)$ converges to $u$ in the following sense:

$$
u_{n} \longrightarrow u \quad \text { strongly in } C([0, T] ; H)
$$

and the limit $u$ becomes the unique weak solution of $\left(\mathrm{CP}\left(\varphi^{t}, f, u_{0}\right)\right)$. Moreover,

$$
\int_{0}^{T} \varphi_{n}^{t}\left(u_{n}(t)\right) d t \longrightarrow \int_{0}^{T} \varphi^{t}(u(t)) d t
$$

In particular, if $\varphi_{n}^{0}\left(u_{0, n}\right)$ is bounded for all $n \in \mathbb{N}$, then the limit $u$ becomes a strong solution of $\left(\mathrm{CP}\left(\varphi^{t}, f, u_{0}\right)\right)$.

2.2. Periodic problem. In this subsection, we consider the following periodic problem $\left(\operatorname{PP}\left(\varphi^{t}, f\right)\right)$ :

$$
\left(\mathrm{PP}\left(\varphi^{t}, f\right)\right)
$$

$$
\begin{gathered}
\frac{d u}{d t}(t)+\partial_{H} \varphi^{t}(u(t)) \ni f(t) \quad \text { in } H, 0<t<T, \\
u(0)=u(T) .
\end{gathered}
$$

We are concerned with strong solutions of $\left(\operatorname{PP}\left(\varphi^{t}, f\right)\right)$ in the following sense.

Definition 2.8. A function $u \in C([0, T] ; H)$ is said to be a strong solution of $\left(\operatorname{PP}\left(\varphi^{t}, f\right)\right)$ if $u$ is a strong solution of $\left(\mathrm{E}\left(\varphi^{t}, f\right)\right)$ such that $u(0)=u(T)$.

To state our results, define

$$
\Psi_{\pi}\left(\alpha, \beta, C_{0}\right):=\left\{\begin{array}{ll}
\left\{\varphi^{t}\right\}_{t \in[0, T]} \in \Psi(\alpha, \beta) ; & |u|_{H}^{2} \leq C_{0}\left(\varphi^{t}(u)+1\right) \forall u \in D\left(\varphi^{t}\right), \forall t \in[0, T], \\
D\left(\varphi^{T}\right) \subset D\left(\varphi^{0}\right)
\end{array}\right\}
$$

for any positive constant $C_{0}$. Moreover, we write $\left\{\varphi^{t}\right\}_{t \in[0, T]} \in B_{\pi}\left(\alpha, \beta, C_{0},\left\{M_{r}\right\}_{r \geq 0}\right)$ if the following hold true.

(i) $\left\{\varphi^{t}\right\}_{t \in[0, T]} \in \Psi_{\pi}\left(\alpha, \beta, C_{0}\right)$.

(ii) There exists a function $h:[0, T] \rightarrow H$ such that (2.9) holds and $h(0)=h(T)$.

(iii) For every $r \in[0,+\infty)$, (2.10) holds.

(iv) $\varphi^{0}(u) \leq \varphi^{T}(u)$ for all $u \in D\left(\varphi^{T}\right)$.

Then it is easily seen that $B_{\pi}\left(\alpha, \beta, C_{0},\left\{M_{r}\right\}_{r \geq 0}\right) \subset B\left(\alpha, \beta, C_{0},\left\{M_{r}\right\}_{r \geq 0}\right)$.

The existence of strong solutions for $\left(\operatorname{PP}\left(\varphi^{t}, f\right)\right)$ is assured by the following. 
914 Convergence of functionals and parabolic equations

Theorem 2.9 [10]. Let $\left\{\varphi^{t}\right\}_{t \in[0, T]} \in \Psi_{\pi}\left(\alpha, \beta, C_{0}\right)$ be such that $\alpha(r, \cdot) \in W^{1,2}(0, T)$, $\beta(r, \cdot) \in W^{1,1}(0, T)$ for all $r \in[0,+\infty)$. Then for all $f \in L^{2}(0, T ; H),\left(\operatorname{PP}\left(\varphi^{t}, f\right)\right)$ has at least one strong solution $u$ satisfying

$$
\frac{d u}{d t} \in L^{2}(0, T ; H), \quad \sup _{t \in[0, T]} \varphi^{t}(u(t))<+\infty .
$$

In particular, if $\varphi^{t}$ is strictly convex on $H$ for a.e. $t \in(0, T)$, then every strong solution of $\left(\mathrm{PP}\left(\varphi^{t}, f\right)\right)$ is unique.

We next focus on the convergence of strong solutions $u_{n}$ for $\left(\operatorname{PP}\left(\varphi_{n}^{t}, f_{n}\right)\right)$ when $\varphi_{n}^{t} \rightarrow$ $\varphi^{t}$ on $H$ in the sense of Mosco and $f_{n} \rightarrow f$ weakly in $L^{2}(0, T ; H)$. However, any studies similar to Theorem 2.7 have not been done on the periodic problem $\left(\operatorname{PP}\left(\varphi_{n}^{t}, f_{n}\right)\right)$ yet, which would be caused by a difficulty peculiar to the periodic problem. More precisely, by virtue of Theorems 2.6 and 2.7, for any $f \in L^{2}(0, T ; H)$ and $u_{0} \in{\overline{D\left(\varphi^{0}\right)}}^{H}$, every unique weak solution of $\left(\mathrm{CP}\left(\varphi^{t}, f, u_{0}\right)\right)$ becomes the limit of unique weak solutions $u_{n}$ for $\left(\mathrm{CP}\left(\varphi_{n}^{t}, f, u_{0}\right)\right)$ as $n \rightarrow+\infty$. However, in general, periodic solutions could not be unique. Hence there could exist a strong solution $u$ of $\left(\operatorname{PP}\left(\varphi^{t}, f\right)\right)$ such that any strong solutions $u_{n}$ of $\left(\operatorname{PP}\left(\varphi_{n}^{t}, f\right)\right)$ never converge to $u$ as $n \rightarrow+\infty$. In fact, we can give such a counter example (see Remark 3.10).

Thus because of the essential difference described above, the strong convergence of solutions $u_{n}$ for $\left(\operatorname{PP}\left(\varphi_{n}^{t}, f_{n}\right)\right)$ in $C([0, T] ; H)$ cannot be verified by the same manner as in the case of the Cauchy problem (see the proof of [10, Theorem 2.7.1]); so in order to cope with this difficulty, we introduce the following level set compactness assumption on $\left\{\varphi_{n}^{t}\right\}_{n \in \mathbb{N}}$.

(A1) For every $\lambda>0$ and $t \in[0, T]$, any sequence $\left(u_{n}\right)$ in $H$ satisfying $\sup _{n \in \mathbb{N}}\left\{\varphi_{n}^{t}\left(u_{n}\right)+\right.$ $\left.\left|u_{n}\right|_{H}\right\} \leq \lambda$ is precompact in $H$.

Then our result can be stated as follows.

Theorem 2.10. For every $n \in \mathbb{N}$, let $\left\{\varphi_{n}^{t}\right\}_{t \in[0, T]} \in B_{\pi}\left(\alpha_{n}, \beta_{n}, C_{0},\left\{M_{r}\right\}_{r \geq 0}\right)$ and let $\left\{\varphi^{t}\right\}_{t \in[0, T]} \in \Psi(\alpha, \beta)$ be such that $\alpha_{n}(r, \cdot), \alpha(r, \cdot) \in W^{1,2}(0, T)$ and $\beta_{n}(r, \cdot), \beta(r, \cdot) \in$ $W^{1,1}(0, T)$ for every $r \in[0,+\infty)$. Suppose that $\varphi_{n}^{t} \rightarrow \varphi^{t}$ on $H$ in the sense of Mosco as $n \rightarrow+\infty$ and that $(A 1)$ holds. Moreover, let $\left(f_{n}\right)$ be a sequence in $L^{2}(0, T ; H)$ such that $f_{n} \rightarrow f$ weakly in $L^{2}(0, T ; H)$ and let $\left(u_{n}\right)$ be a sequence of strong solutions for $\left(\operatorname{PP}\left(\varphi_{n}^{t}, f_{n}\right)\right)$. Then there exists a subsequence $\left(n_{k}\right)$ of $(n)$ such that $u_{n_{k}}$ converges to $u$ in the following sense:

$$
u_{n_{k}} \longrightarrow u \quad \text { strongly in } C([0, T] ; H) \text {, weakly in } W^{1,2}(0, T ; H) \text {, }
$$

and the limit $u$ becomes a strong solution of $\left(\mathrm{PP}\left(\varphi^{t}, f\right)\right)$. Moreover,

$$
\int_{0}^{T} \varphi_{n}^{t}\left(u_{n}(t)\right) d t \longrightarrow \int_{0}^{T} \varphi^{t}(u(t)) d t .
$$

Remark 2.11. (1) In Theorem 2.10, the limit $u$ possibly depends on the choice of the subsequence $\left(n_{k}\right)$. 
(2) By virtue of the assumptions on $\left\{\varphi_{n}^{t}\right\}_{t \in[0, T]}$ and $\left\{\varphi^{t}\right\}_{t \in[0, T]}$ in Theorem 2.10, we can verify that $\left\{\varphi^{t}\right\}_{t \in[0, T]} \in \Psi_{\pi}\left(\alpha, \beta, C_{0}\right)$. Indeed, let $u \in D\left(\varphi^{T}\right)$. Then we can take a sequence $\left(u_{n}\right)$ in $H$ such that $u_{n} \rightarrow u$ strongly in $H$ and $\varphi_{n}^{T}\left(u_{n}\right) \rightarrow \varphi^{T}(u)$. Moreover, from the fact that $\varphi_{n}^{0} \leq \varphi_{n}^{T}$, it follows that

$$
\varphi_{n}^{0}\left(u_{n}\right) \leq \varphi_{n}^{T}\left(u_{n}\right) \longrightarrow \varphi^{T}(u)
$$

Furthermore, since $\liminf _{n \rightarrow+\infty} \varphi_{n}^{0}\left(u_{n}\right) \geq \varphi^{0}(u)$, we have $\varphi^{0}(u) \leq \varphi^{T}(u)$, which implies $D\left(\varphi^{T}\right) \subset D\left(\varphi^{0}\right)$. Similarly we can also deduce that $|u|_{H}^{2} \leq C_{0}\left(\varphi^{t}(u)+1\right)$ for all $u \in D\left(\varphi^{t}\right)$ and $t \in[0, T]$.

Proof of Theorem 2.10. Since $\left\{\varphi_{n}^{t}\right\}_{t \in[0, T]} \in B_{\pi}\left(\alpha_{n}, \beta_{n}, C_{0},\left\{M_{r}\right\}_{r \geq 0}\right)$ for all $n \in \mathbb{N}$, we can take a sequence $\left(h_{n}\right)$ such that

$$
\begin{gathered}
h_{n}(0)=h_{n}(T) \\
\sup _{t \in[0, T]}\left\{\left|h_{n}(t)\right|_{H}+\left|\varphi_{n}^{t}\left(h_{n}(t)\right)\right|\right\}+\left(\int_{0}^{T}\left|\frac{d h_{n}}{d t}(t)\right|_{H}^{2} d t\right)^{1 / 2} \leq C_{0} .
\end{gathered}
$$

Moreover, since $\left\{\varphi_{n}^{t}\right\}_{t \in[0, T]} \in \Psi_{\pi}\left(\alpha_{n}, \beta_{n}, C_{0}\right)$ for all $n \in \mathbb{N}$, we see that

$$
|u|_{H}^{2} \leq C_{0}\left(\varphi_{n}^{t}(u)+1\right) \quad \forall u \in D\left(\varphi_{n}^{t}\right), \forall t \in[0, T], \forall n \in \mathbb{N} .
$$

Now let $u_{n}$ be a strong solution of $\left(\operatorname{PP}\left(\varphi_{n}^{t}, f_{n}\right)\right)$ for each $n \in \mathbb{N}$. Then multiplying the inclusion in $\left(\operatorname{PP}\left(\varphi_{n}^{t}, f_{n}\right)\right)$ by $u_{n}(t)-h_{n}(t)$, we have

$$
\begin{aligned}
\frac{1}{2} \frac{d}{d t} & \left|u_{n}(t)-h_{n}(t)\right|_{H}^{2}+\varphi_{n}^{t}\left(u_{n}(t)\right) \\
& \leq \varphi_{n}^{t}\left(h_{n}(t)\right)+\left(f_{n}(t)-\frac{d h_{n}}{d t}(t), u_{n}(t)-h_{n}(t)\right)_{H} \\
& \leq C_{0}+\left(\left|f_{n}(t)\right|_{H}+\left|\frac{d h_{n}}{d t}(t)\right|_{H}\right)\left|u_{n}(t)-h_{n}(t)\right|_{H} .
\end{aligned}
$$

Now by (2.26) it follows that

$$
\begin{aligned}
& \frac{1}{2} \frac{d}{d t}\left|u_{n}(t)-h_{n}(t)\right|_{H}^{2}+\alpha\left|u_{n}(t)-h_{n}(t)\right|_{H}^{2} \\
& \quad \leq C\left(\left|h_{n}(t)\right|_{H}^{2}+1\right)+\left(\left|f_{n}(t)\right|_{H}+\left|\frac{d h_{n}}{d t}(t)\right|_{H}\right)\left|u_{n}(t)-h_{n}(t)\right|_{H}
\end{aligned}
$$

for some $\alpha>0$. Hence by [11, Lemma 4.2], we get, by (2.25),

$$
\sup _{t \in[0, T]}\left|u_{n}(t)-h_{n}(t)\right|_{H} \leq C,
$$

which implies

$$
\sup _{t \in[0, T]}\left|u_{n}(t)\right|_{H} \leq C_{1}
$$


916 Convergence of functionals and parabolic equations

Furthermore, integrating (2.27) over $(0, T)$, we have

$$
\int_{0}^{T} \varphi_{n}^{t}\left(u_{n}(t)\right) d t \leq C_{2}
$$

Hence by (2.31) there exists $t_{n} \in(0, T)$ such that $\varphi_{n}^{t_{n}}\left(u_{n}\left(t_{n}\right)\right) \leq C_{2} / T$.

Next multiplying the inclusion in $\left(\operatorname{PP}\left(\varphi_{n}^{t}, f_{n}\right)\right)$ by $d u_{n}(t) / d t$, we have

$$
\begin{aligned}
& \left|\frac{d u_{n}}{d t}(t)\right|_{H}^{2}+\left(g_{n}(t), \frac{d u_{n}}{d t}(t)\right)_{H} \\
& \quad=\left(f_{n}(t), \frac{d u_{n}}{d t}(t)\right)_{H} \leq\left|f_{n}(t)\right|_{H}^{2}+\frac{1}{4}\left|\frac{d u_{n}}{d t}(t)\right|_{H}^{2},
\end{aligned}
$$

where $g_{n}(t):=f_{n}(t)-d u_{n}(t) / d t \in \partial_{H} \varphi_{n}^{t}\left(u_{n}(t)\right)$. Hence put $r_{0}=C_{1}$. Then by [12, Lemma 2.4], it follows from (2.30) that

$$
\begin{aligned}
& \left|\left(g_{n}(t), \frac{d u_{n}}{d t}(t)\right)_{H}-\frac{d}{d t} \varphi_{n}^{t}\left(u_{n}(t)\right)\right| \\
& \quad \leq\left|\dot{\alpha}_{n}\left(r_{0}, t\right)\right|\left|g_{n}(t)\right|_{H}\left\{\left|\varphi_{n}^{t}\left(u_{n}(t)\right)\right|+1\right\}^{1 / 2}+\left|\dot{\beta}_{n}\left(r_{0}, t\right)\right|\left\{\left|\varphi_{n}^{t}\left(u_{n}(t)\right)\right|+1\right\}
\end{aligned}
$$

for a.e. $t \in(0, T)$. Thus

$$
\begin{aligned}
& \frac{3}{4}\left|\frac{d u_{n}}{d t}(t)\right|_{H}^{2}+\frac{d}{d t} \varphi_{n}^{t}\left(u_{n}(t)\right) \\
& \leq\left|f_{n}(t)\right|_{H}^{2}+\left|\dot{\alpha}_{n}\left(r_{0}, t\right)\right|\left|f_{n}(t)-\frac{d u_{n}}{d t}(t)\right|_{H}\left\{\left|\varphi_{n}^{t}\left(u_{n}(t)\right)\right|+1\right\}^{1 / 2} \\
& \quad+\left|\dot{\beta}_{n}\left(r_{0}, t\right)\right|\left\{\left|\varphi_{n}^{t}\left(u_{n}(t)\right)\right|+1\right\} \\
& \leq \frac{5}{4}\left|f_{n}(t)\right|_{H}^{2}+\frac{1}{4}\left|\frac{d u_{n}}{d t}(t)\right|_{H}^{2} \\
&+\left\{2\left|\dot{\alpha}_{n}\left(r_{0}, t\right)\right|^{2}+\left|\dot{\beta}_{n}\left(r_{0}, t\right)\right|\right\}\left\{\left|\varphi_{n}^{t}\left(u_{n}(t)\right)\right|+1\right\} .
\end{aligned}
$$

Integrating (2.34) over $\left(t_{n}, t\right)$ and noting that (2.26) implies $\left|\varphi_{n}^{t}(u)\right| \leq \varphi_{n}^{t}(u)+2$ for all $u \in D\left(\varphi_{n}^{t}\right)$, we observe

$$
\begin{aligned}
\frac{1}{2} \int_{t_{n}}^{t} \mid & \left.\frac{d u_{n}}{d \tau}(\tau)\right|_{H} ^{2} d \tau+\varphi_{n}^{t}\left(u_{n}(t)\right) \\
\leq & \varphi_{n}^{t_{n}}\left(u_{n}\left(t_{n}\right)\right)+\frac{5}{4} \int_{0}^{T}\left|f_{n}(\tau)\right|_{H}^{2} d \tau \\
& \quad+\int_{t_{n}}^{t}\left\{2\left|\dot{\alpha}_{n}\left(r_{0}, \tau\right)\right|^{2}+\left|\dot{\beta}_{n}\left(r_{0}, \tau\right)\right|\right\}\left\{\varphi_{n}^{\tau}\left(u_{n}(\tau)\right)+3\right\} d \tau
\end{aligned}
$$


for all $t \in\left[t_{n}, T\right]$. Thus from the fact that

$$
\begin{gathered}
\int_{0}^{T}\left|\dot{\alpha}_{n}\left(r_{0}, \tau\right)\right|^{2} d \tau+\int_{0}^{T}\left|\dot{\beta}_{n}\left(r_{0}, \tau\right)\right| d \tau \leq M_{r_{0}}, \\
\int_{0}^{T}\left|f_{n}(\tau)\right|_{H}^{2} d \tau \leq C, \quad \varphi_{n}^{t_{n}}\left(u_{n}\left(t_{n}\right)\right) \leq \frac{C_{2}}{T},
\end{gathered}
$$

by Gronwall's inequality, it follows that

$$
\sup _{t \in\left[t_{n}, T\right]} \varphi_{n}^{t}\left(u_{n}(t)\right) \leq C_{3}
$$

Hence since $u_{n}(0)=u_{n}(T)$ and $\varphi_{n}^{0}(u) \leq \varphi_{n}^{T}(u)$ for all $u \in D\left(\varphi_{n}^{T}\right)$ and $n \in \mathbb{N}$, we find that $\varphi_{n}^{0}\left(u_{n}(0)\right) \leq \varphi_{n}^{T}\left(u_{n}(T)\right) \leq C_{3}$. Moreover, integrating (2.34) over $(0, t)$, we also get

$$
\begin{aligned}
\frac{1}{2} \int_{0}^{t} \mid & \left.\frac{d u_{n}}{d \tau}(\tau)\right|_{H} ^{2} d \tau+\varphi_{n}^{t}\left(u_{n}(t)\right) \\
\leq & \varphi_{n}^{0}\left(u_{n}(0)\right)+\frac{5}{4} \int_{0}^{T}\left|f_{n}(\tau)\right|_{H}^{2} d \tau \\
& +\int_{0}^{t}\left\{2\left|\dot{\alpha}_{n}\left(r_{0}, \tau\right)\right|^{2}+\left|\dot{\beta}_{n}\left(r_{0}, \tau\right)\right|\right\}\left\{\varphi_{n}^{\tau}\left(u_{n}(\tau)\right)+3\right\} d \tau
\end{aligned}
$$

for all $t \in[0, T]$. Thus Gronwall's inequality implies

$$
\sup _{t \in[0, T]} \varphi_{n}^{t}\left(u_{n}(t)\right) \leq C
$$

Moreover, it follows from (2.26), (2.38), and (2.39) that

$$
\int_{0}^{T}\left|\frac{d u_{n}}{d t}(t)\right|_{H}^{2} d t \leq C .
$$

Hence by $\left(\operatorname{PP}\left(\varphi_{n}^{t}, f_{n}\right)\right),(2.40)$ implies

$$
\int_{0}^{T}\left|g_{n}(t)\right|_{H}^{2} d t \leq C .
$$

By virtue of the above a priori estimates, we can take a subsequence $\left(n_{k}\right)$ of $(n)$ such that the following convergences hold true:

$$
\begin{array}{ll}
u_{n_{k}} \longrightarrow u & \text { weakly in } W^{1,2}(0, T ; H), \\
g_{n_{k}} \longrightarrow g & \text { weakly in } L^{2}(0, T ; H) .
\end{array}
$$

Moreover, by (A1), it follows from (2.30) and (2.39) that

$$
\left(u_{n}(t)\right) \text { is precompact in } H \quad \forall t \in[0, T] .
$$


918 Convergence of functionals and parabolic equations

Furthermore, by $(2.40)$, we can deduce that $u_{n}$ is equicontinuous in $C([0, T] ; H)$ for all $n \in \mathbb{N}$. Thus Ascoli's theorem implies

$$
u_{n_{k}} \longrightarrow u \quad \text { strongly in } C([0, T] ; H)
$$

for a suitable subsequence $\left(n_{k}\right)$ of $(n)$. Hence since $u_{n}(0)=u_{n}(T)$ for all $n \in \mathbb{N}$, we have $u(0)=u(T)$.

In the rest of this proof, we write $n$ simply for $n_{k}$. Now define $\Phi^{T}$ and $\Phi_{n}^{T}$ as in (2.11) with obvious replacements and let $v \in D\left(\Phi^{T}\right)$ be fixed. Then by Proposition 2.4 we can take a sequence $\left(v_{n}\right)$ in $\mathscr{H}_{T}:=L^{2}(0, T ; H)$ such that

$$
v_{n} \longrightarrow v \quad \text { strongly in } \mathcal{H}_{T}, \quad \Phi_{n}^{T}\left(v_{n}\right) \longrightarrow \Phi^{T}(v) .
$$

Now since $g_{n} \in \partial_{\mathscr{H}_{T}} \Phi_{n}^{T}\left(u_{n}\right)$, we have

$$
\begin{gathered}
\int_{0}^{T}\left(f_{n}(t)-\frac{d u_{n}}{d t}(t), u_{n}(t)-v_{n}(t)\right)_{H} d t \\
\quad=\int_{0}^{T}\left(g_{n}(t), u_{n}(t)-v_{n}(t)\right)_{H} d t \\
\geq \Phi_{n}^{T}\left(u_{n}\right)-\Phi_{n}^{T}\left(v_{n}\right) .
\end{gathered}
$$

Moreover, by Proposition 2.4, it follows from (2.45) that

$$
\liminf _{n \rightarrow+\infty} \Phi_{n}^{T}\left(u_{n}\right) \geq \Phi^{T}(u)
$$

Thus passing to the limit $n \rightarrow+\infty$ in (2.47), by (2.42) and (2.45), we find

$$
\int_{0}^{T}\left(f(t)-\frac{d u}{d t}(t), u(t)-v(t)\right)_{H} d t \geq \Phi^{T}(u)-\Phi^{T}(v)
$$

which together with the arbitrariness of $v \in D\left(\Phi^{T}\right)$ implies $u \in D\left(\partial_{\mathscr{H}_{T}} \Phi^{T}\right)$ and $g=f-$ $d u / d t \in \partial_{\mathscr{H}_{T}} \Phi^{T}(u)$. Hence by [9, Proposition 1.1], we deduce that $g(t) \in \partial_{H} \varphi^{t}(u(t))$ for a.e. $t \in(0, T)$. Therefore $u$ is a strong solution of $\left(\operatorname{PP}\left(\varphi^{t}, f\right)\right)$.

Finally we prove (2.22). Since $u \in D\left(\Phi^{T}\right)$, by Proposition 2.4, we can take a sequence $\left(w_{n}\right)$ in $\mathscr{H}_{T}$ such that $w_{n} \rightarrow u$ strongly in $\mathscr{H}_{T}$ and $\Phi_{n}^{T}\left(w_{n}\right) \rightarrow \Phi^{T}(u)$. Hence we get, by (2.40) and (2.45),

$$
\begin{aligned}
& \Phi_{n}^{T}\left(u_{n}\right)-\Phi_{n}^{T}\left(w_{n}\right) \\
& \quad \leq \int_{0}^{T}\left(f_{n}(t)-\frac{d u_{n}}{d t}(t), u_{n}(t)-w_{n}(t)\right)_{H} d t \\
& \quad \leq C\left\{\int_{0}^{T}\left|u_{n}(t)-u(t)\right|_{H}^{2} d t+\int_{0}^{T}\left|w_{n}(t)-u(t)\right|_{H}^{2} d t\right\}^{1 / 2} \longrightarrow 0,
\end{aligned}
$$


which implies

$$
\limsup _{n \rightarrow+\infty} \Phi_{n}^{T}\left(u_{n}\right) \leq \Phi^{T}(u)
$$

Therefore combining (2.48) and (2.51), we can derive (2.22).

\section{Applications to parabolic equations}

The first application is concerned with quasilinear parabolic equations associated with the $p$-Laplacian such as

$$
\frac{\partial u_{p}}{\partial t}(x, t)-\Delta_{p} u_{p}(x, t)=f(x, t), \quad(x, t) \in \Omega \times(0, T),
$$

where $\Omega$ denotes a domain in $\mathbb{R}^{N}$ with smooth boundary $\partial \Omega$. The asymptotic behavior of solutions $u_{p}$ to the initial-boundary value problem for (3.1) as $p \rightarrow+\infty$ has already been studied by several authors (see, e.g., $[2,4]$ ). We here generalize (3.1) as follows:

$$
\frac{\partial u_{p}}{\partial t}(x, t)-\Delta_{p}^{\gamma} u_{p}(x, t)=f_{p}(x, t), \quad(x, t) \in \Omega \times(0, T)
$$

where $f_{p} \rightarrow f$ strongly in $L^{2}\left(0, T ; L^{2}(\Omega)\right)$ as $p \rightarrow+\infty$ and $\Delta_{p}^{\gamma}$ is defined by

$$
\Delta_{p}^{\gamma} u(x):=\nabla \cdot\left\{\left(\frac{1}{\gamma(x, t)}\right)^{p}|\nabla u(x)|^{p-2} \nabla u(x)\right\}
$$

for some function $\gamma: \Omega \times(0, T) \rightarrow \mathbb{R}$. This generalization is motivated by some macroscopic model for type-II superconductors (see [1]).

In Section 3.1, we discuss the existence and uniqueness of solutions $u_{p}$ for $\left((\mathrm{P})_{p}\right)$ and the asymptotic behavior of $u_{p}$ as $p \rightarrow+\infty$ for the periodic problem as well as the initialboundary value problem. Solutions of $\left((\mathrm{P})_{p}\right)$ are defined as follows.

Definition 3.1. A function $u \in C\left([0, T] ; L^{2}(\Omega)\right)$ is said to be a strong solution of $\left((\mathrm{P})_{p}\right)$ if the following are both satisfied:

(i) $u(\cdot, t)$ is an $L^{2}(\Omega)$-valued absolutely continuous function on $[0, T]$;

(ii) $u(\cdot, t) \in W_{0}^{1, p}(\Omega)$ for a.e. $t \in(0, T)$ and

$$
\begin{gathered}
\int_{\Omega} \frac{\partial u}{\partial t}(x, t) \phi(x) d x+\int_{\Omega}\left(\frac{1}{\gamma(x, t)}\right)^{p}|\nabla u|^{p-2} \nabla u(x, t) \cdot \nabla \phi(x) d x \\
=\int_{\Omega} f_{p}(x, t) \phi(x) d x
\end{gathered}
$$

for all $\phi \in W_{0}^{1, p}(\Omega)$ and a.e. $t \in(0, T)$.

Moreover, a function $u \in C\left([0, T] ; L^{2}(\Omega)\right)$ is said to be a weak solution of $\left((\mathrm{P})_{p}\right)$ if there exist sequences $\left(f_{p, n}\right) \subset L^{1}\left(0, T ; L^{2}(\Omega)\right)$ and $\left(u_{n}\right) \subset C\left([0, T] ; L^{2}(\Omega)\right)$ such that $u_{n}$ is a strong solution of $\left((\mathrm{P})_{p}\right), f_{p, n} \rightarrow f_{p}$ strongly in $L^{1}\left(0, T ; L^{2}(\Omega)\right)$, and $u_{n} \rightarrow u$ strongly in $C\left([0, T] ; L^{2}(\Omega)\right)$ as $n \rightarrow+\infty$. 
The second application is for the porous medium equation

$$
\frac{\partial u_{m}}{\partial t}(x, t)-\Delta\left|u_{m}\right|^{m-2} u_{m}(x, t)=0, \quad(x, t) \in \Omega \times(0, T) .
$$

Bénilan and Crandall [6] studied the asymptotic behavior of solutions $u_{n}$ to the initialboundary value problem for

$$
\frac{\partial u_{n}}{\partial t}(x, t)-\Delta \varphi_{n}\left(u_{n}(x, t)\right)=0, \quad(x, t) \in \mathbb{R}^{N} \times(0, T),
$$

where $\varphi_{n}$ is a maximal monotone function from $\mathbb{R}$ into itself, when $\varphi_{n} \rightarrow \varphi$ in a proper sense as $n \rightarrow+\infty$. Moreover, their results cover (3.4) for the case where $\Omega=\mathbb{R}^{N}$.

In Section 3.2, we deal with the following.

$\left((\mathrm{PM})_{m}\right)$

$$
\frac{\partial u_{m}}{\partial t}(x, t)-\Delta \beta_{m}\left(x, t, u_{m}(x, t)\right)=f_{m}(x, t), \quad(x, t) \in \Omega \times(0, T)
$$

where

$$
\beta_{m}(x, t, r):=\frac{|r|^{m-2} r}{\gamma(x, t)^{m}} \quad \forall(x, t) \in \Omega \times(0, T), \forall r \in \mathbb{R} .
$$

We then define solutions for $\left((\mathrm{PM})_{m}\right)$ in the following sense.

Definition 3.2. A function $u \in C\left([0, T] ; H^{-1}(\Omega)\right)$ is said to be a strong solution of $\left((\mathrm{PM})_{m}\right)$ if the following are both satisfied:

(i) $u(\cdot, t)$ is an $H^{-1}(\Omega)$-valued absolutely continuous function on $[0, T]$;

(ii) $u(\cdot, t) \in L^{m}(\Omega), \beta_{m}(\cdot, t, u(\cdot, t)) \in H_{0}^{1}(\Omega)$, and

$$
\left\langle\frac{\partial u}{\partial t}(\cdot, t), \phi\right\rangle_{H_{0}^{1}(\Omega)}+\int_{\Omega} \nabla \beta_{m}(x, t, u(x, t)) \cdot \nabla \phi(x) d x=\left\langle f_{m}(\cdot, t), \phi\right\rangle_{H_{0}^{1}(\Omega)}
$$

for all $\phi \in H_{0}^{1}(\Omega)$ and a.e. $t \in(0, T)$.

Moreover, a function $u \in C\left([0, T] ; H^{-1}(\Omega)\right)$ is said to be a weak solution of $\left((\mathrm{PM})_{m}\right)$ if there exist sequences $\left(f_{m, n}\right) \subset L^{1}\left(0, T ; H^{-1}(\Omega)\right)$ and $\left(u_{n}\right) \subset C\left([0, T] ; H^{-1}(\Omega)\right)$ such that $u_{n}$ is a strong solution of $\left((\mathrm{PM})_{m}\right), f_{m, n} \rightarrow f_{m}$ strongly in $L^{1}\left(0, T ; H^{-1}(\Omega)\right)$, and $u_{n} \rightarrow u$ strongly in $C\left([0, T] ; H^{-1}(\Omega)\right)$ as $n \rightarrow+\infty$.

We also investigate the existence and uniqueness of solutions $u_{m}$ for $\left((\mathrm{PM})_{m}\right)$ and the asymptotic behavior of $u_{m}$ as $m \rightarrow+\infty$ for the periodic problem as well as the initialboundary value problem. 


\subsection{Asymptotic behavior of solutions for parabolic equations associated with $p$-Laplacian as $p \rightarrow+\infty$}

Problem 3.3. Find a unique solution $u_{p}$ of the initial-boundary value problem for $\left((\mathrm{P})_{p}\right)$ with the boundary condition $u_{p}(x, t)=0,(x, t) \in \partial \Omega \times(0, T)$, and the initial condition $u_{p}(x, 0)=u_{0, p}(x), x \in \Omega$, which is denoted by (IBVP1) , and investigate the asymptotic behavior of $u_{p}$ as $p \rightarrow+\infty$.

To this end, we introduce the following hypotheses:

(H1) $\Omega$ is a bounded domain in $\mathbb{R}^{N}$,

$$
\begin{aligned}
& \gamma(x, t)=\pi(x) \phi(t), \quad \pi \in L^{\infty}(\Omega), \phi \in W^{1,2}(0, T), \\
& \gamma(x, t) \geq \delta_{0}>0 \quad \text { for a.e. } x \in \Omega \text { and all } t \in[0, T] .
\end{aligned}
$$

Moreover, set $H:=L^{2}(\Omega)$ and define $\varphi_{p}^{t}: H \rightarrow[0,+\infty]$ as follows:

$$
\varphi_{p}^{t}(u):= \begin{cases}\frac{1}{p} \int_{\Omega}\left(\frac{|\nabla u(x)|}{\gamma(x, t)}\right)^{p} d x & \text { if } u \in W_{0}^{1, p}(\Omega), \frac{\nabla u}{\gamma(\cdot, t)} \in\left(L^{p}(\Omega)\right)^{N} \\ +\infty & \text { otherwise. }\end{cases}
$$

Then (H1) implies

$$
\varphi_{p}^{t} \in \Psi(H), \quad D\left(\varphi_{p}^{t}\right)=W_{0}^{1, p}(\Omega) \quad \forall t \in[0, T] .
$$

Moreover, $\partial_{H} \varphi_{p}^{t}(u)$ coincides with $-\Delta_{p}^{\gamma} u$ with the homogeneous Dirichlet boundary condition $\left.u\right|_{\partial \Omega}=0$ in the distribution sense. Hence by Definition 3.1, (IBVP1) $p$ is equivalent to $\left(\mathrm{CP}\left(\varphi_{p}^{t}, f_{p}, u_{0, p}\right)\right)$. lows.

As for the existence of a unique solution to $\left(\mathrm{CP}\left(\varphi_{p}^{t}, f, u_{0}\right)\right)$, our result is stated as fol-

Theorem 3.4. Suppose that (H1) is satisfied and let $p \in(1,+\infty)$. Then for all $f \in L^{1}(0, T$; $\left.L^{2}(\Omega)\right)$ and $u_{0} \in L^{2}(\Omega),\left(\mathrm{CP}\left(\varphi_{p}^{t}, f, u_{0}\right)\right)$ has a unique weak solution $u_{p}$. In particular, if $f \in L^{2}\left(0, T ; L^{2}(\Omega)\right)$ and $u_{0} \in W_{0}^{1, p}(\Omega)$, the weak solution $u_{p}$ becomes a strong solution of $\left(\mathrm{CP}\left(\varphi_{p}^{t}, f, u_{0}\right)\right)$.

Proof of Theorem 3.4. We first claim that $\left\{\varphi_{p}^{t}\right\}_{t \in[0, T]} \in \Psi\left(\alpha_{1}, 0\right)$ for some function $\alpha_{1}$ : $[0,+\infty) \times[0, T] \rightarrow \mathbb{R}$. Indeed, let $t_{0} \in[0, T]$ and $u_{0} \in D\left(\varphi_{p}^{t_{0}}\right)$ be fixed and define the function $u:[0, T] \rightarrow H$ as follows:

$$
u(t):=\frac{\phi(t)}{\phi\left(t_{0}\right)} u_{0} \in D\left(\varphi_{p}^{t}\right) \quad \forall t \in[0, T] .
$$

Since (H1) says $\gamma(x, t)=\pi(x) \phi(t)$, it follows that

$$
\nabla u(t)=\frac{\phi(t)}{\phi\left(t_{0}\right)} \nabla u_{0}=\frac{\gamma(x, t)}{\gamma\left(x, t_{0}\right)} \nabla u_{0} \quad \forall t \in[0, T],
$$


922 Convergence of functionals and parabolic equations

which implies

$$
\varphi_{p}^{t}(u(t))=\frac{1}{p} \int_{\Omega}\left(\frac{|\nabla u(x, t)|}{\gamma(x, t)}\right)^{p} d x=\frac{1}{p} \int_{\Omega}\left(\frac{\left|\nabla u_{0}(x)\right|}{\gamma\left(x, t_{0}\right)}\right)^{p} d x=\varphi_{p}^{t_{0}}\left(u_{0}\right) .
$$

Moreover, we see

$$
\begin{aligned}
\left|u(t)-u_{0}\right|_{H} & =\left|\frac{\phi(t)}{\phi\left(t_{0}\right)}-1\right|\left|u_{0}\right|_{H} \\
& \leq \frac{1}{\delta_{0}}|\pi|_{L^{\infty}(\Omega)}\left|\phi(t)-\phi\left(t_{0}\right)\right|\left|u_{0}\right|_{H} \\
& \leq\left|\alpha_{1}(r, t)-\alpha_{1}\left(r, t_{0}\right)\right| \quad \forall r \geq\left|u_{0}\right|_{H},
\end{aligned}
$$

where $\alpha_{1}$ is given by

$$
\alpha_{1}(r, t)=\frac{r}{\delta_{0}}|\pi|_{L^{\infty}(\Omega)} \phi(t) \in W^{1,2}(0, T)
$$

Therefore we conclude that $\left\{\varphi_{p}^{t}\right\}_{t \in[0, T]} \in \Psi\left(\alpha_{1}, 0\right)$ for every $p \in(1,+\infty)$. Then applying Theorem 2.6 to $\left(\mathrm{CP}\left(\varphi_{p}^{t}, f, u_{0}\right)\right)$, we can derive the desired result.

Now we are going to describe our result on the asymptotic behavior of $u_{p}$ as $p \rightarrow+\infty$. Theorem 3.5. Suppose that (H1) is satisfied and define

$$
K^{t}:=\left\{u \in H_{0}^{1}(\Omega) ;|\nabla u(x)| \leq \gamma(x, t) \text { for a.e. } x \in \Omega\right\} .
$$

Let $\left(p_{n}\right)$ be a sequence in $(1,+\infty)$ such that $p_{n} \rightarrow+\infty$ as $n \rightarrow+\infty$. Moreover, let $f_{n}, f \in$ $L^{2}\left(0, T ; L^{2}(\Omega)\right), u_{0, n} \in L^{2}(\Omega)$, and $u_{0} \in K^{0}$ be such that

$$
\begin{aligned}
& f_{n} \longrightarrow f \quad \text { strongly in } L^{2}\left(0, T ; L^{2}(\Omega)\right), \\
& u_{0, n} \longrightarrow u_{0} \text { strongly in } L^{2}(\Omega) \text {. }
\end{aligned}
$$

Then the unique weak solution $u_{n}$ of $\left(\mathrm{CP}\left(\varphi_{p_{n}}^{t}, f_{n}, u_{0, n}\right)\right)$ converges to $u$ as $n \rightarrow+\infty$ in the following sense:

$$
u_{n} \longrightarrow u \quad \text { strongly in } C\left([0, T] ; L^{2}(\Omega)\right)
$$

Moreover, the limit $u$ is a unique weak solution of $\left(\mathrm{CP}\left(\varphi_{\infty}^{t}, f, u_{0}\right)\right)$, where $\varphi_{\infty}^{t}$ is defined by

$$
\varphi_{\infty}^{t}(u):= \begin{cases}0 & \text { if } u \in K^{t}, \\ +\infty & \text { if } u \in L^{2}(\Omega) \backslash K^{t} .\end{cases}
$$

In particular, if $\left(1 / p_{n}\right) \int_{\Omega}\left|\nabla u_{0, n}(x)\right|^{p_{n}} d x$ is bounded as $n \rightarrow+\infty$, then the limit $u$ becomes $a$ strong solution of $\left(\mathrm{CP}\left(\varphi_{\infty}^{t}, f, u_{0}\right)\right)$. 
Proof of Theorem 3.5. On account of Theorem 2.7, it suffices to show that

$$
\begin{gathered}
\left\{\varphi_{p_{n}}^{t}\right\}_{t \in[0, T]} \in B\left(\alpha_{1}, 0, C_{0},\left\{M_{r}\right\}_{r \geq 0}\right) \quad \text { for some constants } C_{0},\left\{M_{r}\right\}_{r \geq 0}, \\
\left\{\varphi_{\infty}^{t}\right\}_{t \in[0, T]} \in \Psi\left(\alpha_{1}, 0\right), \\
\varphi_{p_{n}}^{t} \longrightarrow \varphi_{\infty}^{t} \quad \text { on } H \text { in the sense of Mosco as } p_{n} \longrightarrow+\infty
\end{gathered}
$$

We first prove (3.22). We have already seen that $\left\{\varphi_{p}^{t}\right\}_{t \in[0, T]} \in \Psi\left(\alpha_{1}, 0\right)$ for all $p \in$ $(1,+\infty)$. Moreover, it is obvious that $\varphi_{p}^{t} \geq 0$ and that $h \equiv 0$ satisfies

$$
\frac{d h}{d t}(t)=0, \quad \varphi_{p}^{t}(h(t))=0 \quad \forall t \in[0, T], \forall p \in(1,+\infty) .
$$

Hence we can take $C_{0}=0$. Furthermore, we see

$$
\int_{0}^{T}\left|\dot{\alpha}_{1}(r, t)\right|^{2} d t=\left(\frac{r}{\delta_{0}}|\pi|_{L^{\infty}(\Omega)}\right)^{2} \int_{0}^{T}|\dot{\phi}(t)|^{2} d t=: M_{r},
$$

where we note that $M_{r}$ is independent of $p$. Therefore (3.22) holds.

In much the same way as in the proof of Theorem 3.4, we can derive (3.23). Indeed, $u(t)$ appearing in (3.12) satisfies

$$
|\nabla u(x, t)|=\left|\frac{\gamma(x, t)}{\gamma\left(x, t_{0}\right)} \nabla u_{0}(x)\right| \leq \gamma(x, t) \quad \text { for a.e. } x \in \Omega \text { and all } t \in[0, T]
$$

for each $u_{0} \in K^{t_{0}}$ and $t_{0} \in[0, T]$. Hence we deduce that $\varphi_{\infty}^{t}(u(t))=\varphi_{\infty}^{t_{0}}\left(u_{0}\right)=0$ for all $t \in[0, T]$, which together with (3.15) implies $\left\{\varphi_{\infty}^{t}\right\}_{t \in[0, T]} \in \Psi\left(\alpha_{1}, 0\right)$.

Finally (3.24) is derived from the following lemma.

Lemma 3.6. For each $t \in[0, T]$, it follows that

$$
\varphi_{p_{n}}^{t} \longrightarrow \varphi_{\infty}^{t} \quad \text { on } H \text { in the sense of Mosco as } p_{n} \longrightarrow+\infty \text {. }
$$

Proof of Lemma 3.6. Let $t \in[0, T]$ be fixed. We first claim that

$$
\begin{gathered}
\forall u \in D\left(\varphi_{\infty}^{t}\right), \quad \exists\left(u_{n}\right) \subset H ; \\
u_{n} \longrightarrow u \quad \text { strongly in } H, \quad \varphi_{p_{n}}^{t}\left(u_{n}\right) \longrightarrow \varphi_{\infty}^{t}(u) .
\end{gathered}
$$

Indeed, let $u \in D\left(\varphi_{\infty}^{t}\right)=K^{t}$ and put $u_{n}:=u$ for all $n \in \mathbb{N}$. Then since $K^{t} \subset W_{0}^{1, p_{n}}(\Omega)$ for all $n \in \mathbb{N}$, it follows immediately that

$$
\begin{aligned}
0 \leq \varphi_{p_{n}}^{t}\left(u_{n}\right) & =\frac{1}{p_{n}} \int_{\Omega}\left(\frac{|\nabla u(x)|}{\gamma(x, t)}\right)^{p_{n}} d x \\
& \leq \frac{1}{p_{n}}|\Omega| \longrightarrow 0=\varphi_{\infty}^{t}(u) \text { as } p_{n} \longrightarrow+\infty
\end{aligned}
$$

Hence we deduce that (3.29) holds true. 
924 Convergence of functionals and parabolic equations

We next show that

$$
\begin{gathered}
\forall\left(u_{n}\right) \subset H \quad \text { satisfying } u_{n} \longrightarrow u \text { weakly in } H, \\
\liminf _{n \rightarrow+\infty} \varphi_{p_{n}}^{t}\left(u_{n}\right) \geq \varphi_{\infty}^{t}(u) .
\end{gathered}
$$

For the case where $u \in D\left(\varphi_{\infty}^{t}\right)=K^{t}$, it is obvious that $\liminf _{n \rightarrow+\infty} \varphi_{p_{n}}^{t}\left(u_{n}\right) \geq 0=\varphi_{\infty}^{t}(u)$. For the case where $u \notin K^{t}$, we give a proof by contradiction. Suppose that

$$
\exists\left(u_{n}\right) \subset H ; \quad u_{n} \longrightarrow u \quad \text { weakly in } H, \quad \liminf _{n \rightarrow+\infty} \varphi_{p_{n}}^{t}\left(u_{n}\right)<\varphi_{\infty}^{t}(u)=+\infty
$$

Then we can take a subsequence $\left(n^{\prime}\right)$ of $(n)$ such that

$$
\varphi_{p_{n^{\prime}}}^{t}\left(u_{n^{\prime}}\right) \leq C \quad \forall n^{\prime}
$$

which implies

$$
\begin{aligned}
& \left\{\int_{\Omega}\left(\frac{\left|\nabla u_{n^{\prime}}(x)\right|}{\gamma(x, t)}\right)^{p_{n^{\prime}}} d x\right\}^{1 / p_{n^{\prime}}} \\
& \quad \leq\left\{p_{n^{\prime}} \varphi_{p_{n^{\prime}}}^{t}\left(u_{n^{\prime}}\right)\right\}^{1 / p_{n^{\prime}}} \leq\left(p_{n^{\prime}} C\right)^{1 / p_{n^{\prime}}} \longrightarrow 1 \text { as } n^{\prime} \longrightarrow+\infty
\end{aligned}
$$

For simplicity of notation, we write $p$ and $u_{p}$ for $p_{n^{\prime}}$ and $u_{n^{\prime}}$, respectively. Hence by $(\mathrm{H} 1)$ we have

$$
\left(\int_{\Omega}\left|\nabla u_{p}(x)\right|^{p} d x\right)^{1 / p} \leq C
$$

which yields

$$
\begin{aligned}
\left(\int_{\Omega}\left|\nabla u_{p}(x)\right|^{q} d x\right)^{1 / q} & \leq\left(\int_{\Omega}\left|\nabla u_{p}(x)\right|^{p} d x\right)^{1 / p}|\Omega|^{(p-q) /(p q)} \\
& \leq C(|\Omega|+1)^{1 / q} \quad \forall q \in[1, p] .
\end{aligned}
$$

Thus for each $q \in(1,+\infty)$, we can take a subsequence $\left(p_{q}\right)$ of $(p)$ such that

$$
\nabla u_{p_{q}} \longrightarrow \nabla u \quad \text { weakly in }\left(L^{q}(\Omega)\right)^{N}
$$

Here we also observe that $u \in H_{0}^{1}(\Omega)$. In the rest of this proof, we drop $q$ in $p_{q}$. Moreover, by $(\mathrm{H} 1)$, we can also derive

$$
\frac{\nabla u_{p}}{\gamma(\cdot, t)} \longrightarrow \frac{\nabla u}{\gamma(\cdot, t)} \quad \text { weakly in }\left(L^{q}(\Omega)\right)^{N}
$$


Hence it follows from (3.34) and (3.38) that

$$
\begin{aligned}
\left\{\int_{\Omega}\left(\frac{|\nabla u(x)|}{\gamma(x, t)}\right)^{q} d x\right\}^{1 / q} & \leq \liminf _{p \rightarrow+\infty}\left\{\int_{\Omega}\left(\frac{\left|\nabla u_{p}(x)\right|}{\gamma(x, t)}\right)^{q} d x\right\}^{1 / q} \\
& \leq \liminf _{p \rightarrow+\infty}\left\{\int_{\Omega}\left(\frac{\left|\nabla u_{p}(x)\right|}{\gamma(x, t)}\right)^{p} d x\right\}^{1 / p}|\Omega|^{(p-q) /(p q)} \\
& \leq \lim _{p \rightarrow+\infty}(p C)^{1 / p}|\Omega|^{(p-q) /(p q)} \\
& =|\Omega|^{1 / q} .
\end{aligned}
$$

Therefore passing to the limit $q \rightarrow+\infty$, we deduce that

$$
\left|\frac{\nabla u}{\gamma(\cdot, t)}\right|_{L^{\infty}(\Omega)} \leq 1,
$$

which contradicts the fact that $u \notin K^{t}$. Hence (3.31) holds true.

Then by (3.22), (3.23), and (3.24), Theorem 2.7 assures the desired conclusion.

Problem 3.7. Find a solution of the periodic problem for $\left((\mathrm{P})_{p}\right)$ with the boundary condition $u(x, t)=0,(x, t) \in \partial \Omega \times(0, T)$, and the periodic condition $u(x, 0)=u(x, T), x \in$ $\Omega$, which is denoted by $(\mathrm{PP} 1)_{p}$. Moreover, investigate the asymptotic behavior of $u_{p}$ as $p \rightarrow+\infty$.

Just as in Problem 3.3, $(\mathrm{PP} 1)_{p}$ can be also reduced to $\left(\operatorname{PP}\left(\varphi_{p}^{t}, f_{p}\right)\right)$. Then as for the existence of a solution to $\left(\operatorname{PP}\left(\varphi_{p}^{t}, f\right)\right)$, we have the following.

Theorem 3.8. Suppose that (H1) is satisfied and let $p \in[2,+\infty)$. Then for all $f \in L^{2}(0, T$; $\left.L^{2}(\Omega)\right),\left(\operatorname{PP}\left(\varphi_{p}^{t}, f\right)\right)$ has a unique strong solution $u_{p}$.

Proof of Theorem 3.8. We claim that $\left\{\varphi_{p}^{t}\right\}_{t \in[0, T]} \in \Psi_{\pi}\left(\alpha_{1}, 0, C_{0}\right)$ for some positive constant $C_{0}$. Since $H_{0}^{1}(\Omega)$ is continuously embedded in $H$, we see that

$$
\begin{aligned}
|u|_{H}^{2} & \leq C|\nabla u|_{H}^{2} \\
& \leq C|\gamma|_{L^{\infty}(Q)}^{2} \int_{\Omega}\left(\frac{|\nabla u(x)|}{\gamma(x, t)}\right)^{2} d x \\
& \leq C|\gamma|_{L^{\infty}(Q)}^{2}\left\{\frac{2}{p} \int_{\Omega}\left(\frac{|\nabla u(x)|}{\gamma(x, t)}\right)^{p} d x+\frac{p-2}{p}|\Omega|\right\} \\
& \leq 2 C|\gamma|_{L^{\infty}(Q)}^{2}\left\{\varphi_{p}^{t}(u)+|\Omega|\right\} \quad \forall u \in D\left(\varphi_{p}^{t}\right), \forall p \geq 2,
\end{aligned}
$$

where $Q:=\Omega \times[0, T]$. Hence since we have already known that $D\left(\varphi_{p}^{t}\right)=W_{0}^{1, p}(\Omega)$ for all $t \in[0, T]$ and $\left\{\varphi_{p}^{t}\right\}_{t \in[0, T]} \in \Psi\left(\alpha_{1}, 0\right)$ for every $p \in(1,+\infty)$, we deduce that $\left\{\varphi_{p}^{t}\right\}_{t \in[0, T]} \in$ $\Psi_{\pi}\left(\alpha_{1}, 0, C_{0}\right)$ for some positive constant $C_{0}$ independent of $p$. Therefore Theorem 2.9 assures the existence of a strong solution $u_{p}$ for $\left(\operatorname{PP}\left(\varphi_{p}^{t}, f\right)\right)$. Moreover, since $\varphi_{p}^{t}$ is strictly convex on $H$, the periodic solution $u_{p}$ is unique. 
As for the asymptotic behavior of $u_{p}$ as $p \rightarrow+\infty$, we have the following.

Theorem 3.9. Suppose that (H1) is satisfied and that $\gamma(x, 0) \geq \gamma(x, T)$ for a.e. $x \in \Omega$. Let $\left(p_{n}\right)$ be a sequence in $[2,+\infty)$ such that $p_{n} \rightarrow+\infty$ as $n \rightarrow+\infty$ and let $f_{n}, f \in L^{2}\left(0, T ; L^{2}(\Omega)\right)$ be such that

$$
f_{n} \longrightarrow f \quad \text { weakly in } L^{2}\left(0, T ; L^{2}(\Omega)\right)
$$

Then a subsequence $\left(n_{k}\right)$ of $(n)$ can be taken such that the unique strong solution $u_{n_{k}}$ of $\left(\operatorname{PP}\left(\varphi_{p_{n_{k}}}^{t}, f_{n_{k}}\right)\right)$ converges to $u$ as $k \rightarrow+\infty$ in the following sense:

$$
u_{n_{k}} \longrightarrow u \text { strongly in } C\left([0, T] ; L^{2}(\Omega)\right) \text {, weakly in } W^{1,2}\left(0, T ; L^{2}(\Omega)\right) \text {. }
$$

Moreover, the limit $u$ is a strong solution of $\left(\operatorname{PP}\left(\varphi_{\infty}^{t}, f\right)\right)$.

Proof of Theorem 3.9. We first claim that $\left\{\varphi_{p_{n}}^{t}\right\}_{t \in[0, T]} \in B_{\pi}\left(\alpha_{1}, 0, C_{0},\left\{M_{r}\right\}_{r \geq 0}\right)$ for some constants $\left\{M_{r}\right\}_{r \geq 0}$ independent of $n$. Indeed, we have already seen that $\left\{\varphi_{p}^{t}\right\}_{t \in[0, T]} \in$ $\Psi_{\pi}\left(\alpha_{1}, 0, C_{0}\right)$, where $C_{0}$ is a positive constant independent of $p$. Moreover, since $\gamma(x, 0) \geq$ $\gamma(x, T)$ for a.e. $x \in \Omega$, it is obvious that

$$
\varphi_{p}^{0}(u) \leq \varphi_{p}^{T}(u) \quad \forall u \in D\left(\varphi_{p}^{T}\right), \forall p \in(1,+\infty)
$$

The rest of the proof for this claim can be derived as in the proof of Theorem 3.5.

We next prove that $\left\{\varphi_{p_{n}}^{t}\right\}_{n \in \mathbb{N}}$ satisfies (A1). Let $\lambda>0$ and $t \in[0, T]$ be fixed and let $\left(u_{n}\right)$ be a sequence in $H$ such that

$$
\varphi_{p_{n}}^{t}\left(u_{n}\right)+\left|u_{n}\right|_{H} \leq \lambda \quad \forall n \in \mathbb{N} \text {. }
$$

For every $p_{n} \geq 2$, we get

$$
\begin{aligned}
\left(\int_{\Omega}\left|\nabla u_{n}(x)\right|^{2} d x\right)^{1 / 2} & \leq|\gamma(\cdot, t)|_{L^{\infty}(\Omega)}\left\{\int_{\Omega}\left(\frac{\left|\nabla u_{n}(x)\right|}{\gamma(x, t)}\right)^{p_{n}} d x\right\}^{1 / p_{n}}|\Omega|^{\left(p_{n}-2\right) /\left(2 p_{n}\right)} \\
& \leq|\gamma(\cdot, t)|_{L^{\infty}(\Omega)}\left(p_{n} \lambda\right)^{1 / p_{n}}|\Omega|^{\left(p_{n}-2\right) /\left(2 p_{n}\right)} \leq C,
\end{aligned}
$$

where $C$ is a constant independent of $n$. Hence since $H_{0}^{1}(\Omega)$ is compactly embedded in $H$, we deduce that $\left(u_{n}\right)$ becomes precompact in $H$, which implies (A1) with $\varphi_{n}^{t}$ replaced by $\varphi_{p_{n}}^{t}$.

Moreover, Lemma 3.6 says

$$
\varphi_{p_{n}}^{t} \longrightarrow \varphi_{\infty}^{t} \quad \text { on } H \text { in the sense of Mosco. }
$$


Hence by Theorem 2.10 we can take a subsequence $\left(n_{k}\right)$ of $(n)$ such that the unique strong solution $u_{n_{k}}$ of $\left(\operatorname{PP}\left(\varphi_{p_{n_{k}}}^{t}, f_{n_{k}}\right)\right)$ satisfies

$$
u_{n_{k}} \longrightarrow u \quad \text { strongly in } C([0, T] ; H) \text {, weakly in } W^{1,2}(0, T ; H) \text {; }
$$

moreover, $u$ becomes a strong solution of $\left(\operatorname{PP}\left(\varphi_{\infty}^{t}, f\right)\right)$.

Remark 3.10. As mentioned in Theorem 3.8, $\left(\operatorname{PP}\left(\varphi_{p}^{t}, f\right)\right)$ has a unique strong solution. On the other hand, $\left(\operatorname{PP}\left(\varphi_{\infty}^{t}, f\right)\right)$ may have multiple strong solutions. Indeed, let $t_{0} \in[0, T]$ be a minimizer of $\phi$, that is, $0<\phi\left(t_{0}\right) \leq \phi(t)$ for all $t \in[0, T]$. Then we have $K^{t_{0}} \subset K^{t}$ for all $t \in[0, T]$. Hence for every $u_{0} \in K^{t_{0}}, \partial_{H} \varphi_{\infty}^{t}\left(u_{0}\right) \ni 0$ for all $t \in[0, T]$ and $u \equiv u_{0}$ becomes a strong solution for $\left(\operatorname{PP}\left(\varphi_{\infty}^{t}, 0\right)\right)$. Therefore since $K^{t_{0}}$ has infinitely many elements, $\left(\operatorname{PP}\left(\varphi_{\infty}^{t}, 0\right)\right)$ admits infinitely many strong solutions.

Furthermore, since $u_{p} \equiv 0$ is a unique strong solution of $\left(\operatorname{PP}\left(\varphi_{p}^{t}, 0\right)\right)$ for all $p \in(1,+\infty)$, $u_{p}$ never converges to any strong solution $u$ of $\left(\operatorname{PP}\left(\varphi_{\infty}^{t}, 0\right)\right)$ except $u \equiv 0$ as $p \rightarrow+\infty$.

\subsection{Asymptotic behavior of solutions for porous medium equation as $m \rightarrow+\infty$}

Problem 3.11. Find a unique solution $u_{m}$ of the initial-boundary value problem for $\left((\mathrm{PM})_{m}\right)$ with the boundary condition $u(x, t)=0,(x, t) \in \partial \Omega \times(0, T)$, and the initial condition $u(x, 0)=u_{0}(x), x \in \Omega$, which is denoted by (IBVP2 $)_{m}$, and investigate the asymptotic behavior of $u_{m}$ as $m \rightarrow+\infty$.

We will make the following assumptions:

(H2) $\Omega$ is a bounded domain in $\mathbb{R}^{N}$,

$$
\begin{gathered}
\gamma(x, t) \in W^{1,2}\left(0, T ; L^{\infty}(\Omega)\right), \\
\gamma(x, t) \geq \delta_{0}>0 \quad \text { for a.e. } x \in \Omega \text { and all } t \in[0, T] .
\end{gathered}
$$

Set $H:=H^{-1}(\Omega)$ and define $\psi_{m}^{t}: H \rightarrow[0,+\infty]$ as follows:

$$
\psi_{m}^{t}(u):= \begin{cases}\frac{1}{m} \int_{\Omega}\left(\frac{|u(x)|}{\gamma(x, t)}\right)^{m} d x & \text { if } \frac{u}{\gamma(\cdot, t)} \in L^{m}(\Omega), \\ +\infty & \text { otherwise. }\end{cases}
$$

Moreover, we denote $\psi_{m}^{t}$ with $\gamma \equiv 1$ simply by $\psi_{m}$. Then by (H2), we see that

$$
D\left(\psi_{m}^{t}\right)=L^{m}(\Omega) \quad \forall t \in[0, T] .
$$

Now define

$$
(u, v)_{H}:=\left\langle u,\left(-\Delta_{D}\right)^{-1} v\right\rangle_{H_{0}^{1}(\Omega)} \quad \forall u, v \in H,
$$

where $\Delta_{D}$ denotes the Laplacian with the homogeneous Dirichlet boundary condition and $\langle\cdot, \cdot\rangle_{H_{0}^{1}(\Omega)}$ denotes the natural duality pairing between $H_{0}^{1}(\Omega)$ and $H$. 
928 Convergence of functionals and parabolic equations

We now observe that $H_{0}^{1}(\Omega) \subset L^{m^{\prime}}(\Omega)$ for every $m \in[2 N /(N+2),+\infty)$. Hence let $t \in$ $[0, T]$ be fixed and let $[u, f] \in \partial_{H} \psi_{m}^{t}$. We then get, for all $v \in L^{m}(\Omega)$,

$$
\begin{aligned}
\psi_{m}\left(\frac{u}{\gamma(\cdot, t)}\right)-\psi_{m}(v) & =\psi_{m}^{t}(u)-\psi_{m}^{t}(\gamma(\cdot, t) v) \\
& \leq(f, u-\gamma(\cdot, t) v)_{H} \\
& =\left\langle u-\gamma(\cdot, t) v,\left(-\Delta_{D}\right)^{-1} f\right\rangle_{H_{0}^{1}(\Omega)} \\
& =\int_{\Omega} \gamma(x, t)\left(-\Delta_{D}\right)^{-1} f(x)\left(\frac{u(x)}{\gamma(x, t)}-v(x)\right) d x
\end{aligned}
$$

Therefore since $\psi_{m}$ is Fréchet differentiable on $L^{m}(\Omega)$ and its derivative at $u / \gamma(\cdot, t)$ coincides with $|u / \gamma(\cdot, t)|^{m-2} u / \gamma(\cdot, t)$, we can verify

$$
f=\partial_{H} \psi_{m}^{t}(u) \Longleftrightarrow\left(-\Delta_{D}\right)^{-1} f(x)=\beta_{m}(x, t, u(x)) \text { for a.e. } x \in \Omega
$$

for every $m \in[2 N /(N+2),+\infty)$, where we use the maximality of $\partial_{H} \psi_{m}^{t}$. Hence (IBVP2 $)_{m}$ is reduced to $\left(\mathrm{CP}\left(\psi_{m}^{t}, f_{m}, u_{0, m}\right)\right)$.

The existence of solutions is assured by Theorem 2.6.

Theorem 3.12. Suppose that (H2) is satisfied and let $m \in[2,+\infty)$. Then for all $f \in L^{1}(0$, $\left.T ; H^{-1}(\Omega)\right)$ and $u_{0} \in H^{-1}(\Omega),\left(\mathrm{CP}\left(\psi_{m}^{t}, f, u_{0}\right)\right)$ has a unique weak solution $u_{m}$. In particular, if $f \in L^{2}\left(0, T ; H^{-1}(\Omega)\right)$ and $u_{0} \in L^{m}(\Omega)$, the unique weak solution $u_{m}$ becomes a strong solution of $\left(\mathrm{CP}\left(\psi_{m}^{t}, f, u_{0}\right)\right)$.

Proof of Theorem 3.12. By Theorem 2.6, it suffices to verify that $\left\{\psi_{m}^{t}\right\}_{t \in[0, T]} \in \Psi\left(\alpha_{2}, 0\right)$ for some function $\alpha_{2}:[0,+\infty) \times[0, T] \rightarrow \mathbb{R}$. Let $t_{0} \in[0, T]$ and let $u_{0} \in D\left(\psi_{m}^{t_{0}}\right)$ be fixed. Define

$$
u(t):=\frac{\gamma(\cdot, t)}{\gamma\left(\cdot, t_{0}\right)} u_{0} \in L^{m}(\Omega) \quad \forall t \in[0, T]
$$

Then we find that

$$
|u(x, t)|=\gamma(x, t) \frac{\left|u_{0}(x)\right|}{\gamma\left(x, t_{0}\right)} \quad \text { for a.e. } x \in \Omega,
$$

which implies $\psi_{m}^{t}(u(t))=\psi_{m}^{t_{0}}\left(u_{0}\right)$ for all $t \in[0, T]$. Furthermore, for any $\phi \in H_{0}^{1}(\Omega)$, we see that

$$
\begin{aligned}
\left\langle u(t)-u_{0}, \phi\right\rangle_{H_{0}^{1}(\Omega)} & =\int_{\Omega}\left\{\gamma(x, t)-\gamma\left(x, t_{0}\right)\right\} \frac{u_{0}(x)}{\gamma\left(x, t_{0}\right)} \phi(x) d x \\
& \leq\left|\gamma(\cdot, t)-\gamma\left(\cdot, t_{0}\right)\right|_{L^{\infty}(\Omega)}\left|\frac{u_{0}}{\gamma\left(\cdot, t_{0}\right)}\right|_{L^{m}(\Omega)}|\phi|_{L^{m^{\prime}}(\Omega)} .
\end{aligned}
$$

Here since $m \geq 2$, it follows that

$$
|\phi|_{L^{m^{\prime}}(\Omega)} \leq|\phi|_{L^{2}(\Omega)}|\Omega|^{\left(2-m^{\prime}\right) / 2} \leq C|\phi|_{H_{0}^{1}(\Omega)}(|\Omega|+1),
$$


where $C$ is independent of $m$. Thus

$$
\begin{aligned}
\left|u(t)-u_{0}\right|_{H} & \leq C(|\Omega|+1)\left|\gamma(\cdot, t)-\gamma\left(\cdot, t_{0}\right)\right|_{L^{\infty}(\Omega)}\left\{m \psi_{m}^{t_{0}}\left(u_{0}\right)\right\}^{1 / m} \\
& \leq C e^{1 / e}(|\Omega|+1)\left|\gamma(\cdot, t)-\gamma\left(\cdot, t_{0}\right)\right|_{L^{\infty}(\Omega)}\left\{\psi_{m}^{t_{0}}\left(u_{0}\right)+1\right\}^{1 / 2} .
\end{aligned}
$$

Hence it follows that

$$
\left\{\psi_{m}^{t}\right\}_{t \in[0, T]} \in \Psi\left(\alpha_{2}, 0\right)
$$

where $\alpha_{2}$ is given by

$$
\alpha_{2}(r, t)=C e^{1 / e}(|\Omega|+1) \int_{0}^{t}\left|\frac{\partial \gamma}{\partial \tau}(\cdot, \tau)\right|_{L^{\infty}(\Omega)} d \tau \in W^{1,2}(0, T)
$$

As for the asymptotic behavior of $u_{m}$ as $m \rightarrow+\infty$, our result is stated as follows.

Theorem 3.13. Suppose that (H2) is satisfied and define

$$
\kappa^{t}:=\left\{u \in L^{2}(\Omega) ;|u(x)| \leq \gamma(x, t) \text { for a.e. } x \in \Omega\right\} .
$$

Let $\left(m_{n}\right)$ be a sequence in $[2,+\infty)$ such that $m_{n} \rightarrow+\infty$ as $n \rightarrow+\infty$. Moreover, let $f_{n}, f \in$ $L^{2}\left(0, T ; H^{-1}(\Omega)\right), u_{0, n} \in H^{-1}(\Omega)$, and $u_{0} \in \kappa^{0}$ be such that

$$
\begin{aligned}
& f_{n} \longrightarrow f \quad \text { strongly in } L^{2}\left(0, T ; H^{-1}(\Omega)\right) \text {, } \\
& u_{0, n} \longrightarrow u_{0} \quad \text { strongly in } H^{-1}(\Omega) \text {. }
\end{aligned}
$$

Then the unique weak solution $u_{n}$ of $\left(\mathrm{CP}\left(\psi_{m_{n}}^{t}, f_{n}, u_{0, n}\right)\right)$ converges to $u$ as $n \rightarrow+\infty$ in the following sense:

$$
u_{n} \longrightarrow u \quad \text { strongly in } C\left([0, T] ; H^{-1}(\Omega)\right)
$$

Moreover, the limit $u$ is a unique weak solution of $\left(\mathrm{CP}\left(\psi_{\infty}^{t}, f, u_{0}\right)\right)$, where

$$
\psi_{\infty}^{t}(u):= \begin{cases}0 & \text { if } u \in \kappa^{t}, \\ +\infty & \text { if } u \in H^{-1}(\Omega) \backslash \kappa^{t} .\end{cases}
$$

In particular, if $\left(1 / m_{n}\right) \int_{\Omega}\left|u_{0, n}(x)\right|^{m_{n}} d x$ is bounded as $n \rightarrow+\infty$, then the limit $u$ becomes a strong solution of $\left(\mathrm{CP}\left(\psi_{\infty}^{t}, f, u_{0}\right)\right)$.

As in Lemma 3.6, we have the following.

Lemma 3.14. For each $t \in[0, T]$, it follows that

$$
\psi_{m_{n}}^{t} \longrightarrow \psi_{\infty}^{t} \quad \text { on } H \text { in the sense of Mosco as } m_{n} \longrightarrow+\infty
$$


Proof of Lemma 3.14. Let $t \in[0, T]$ be fixed. Then as in the proof of Lemma 3.6, we can easily derive that

$$
\begin{gathered}
\forall u \in D\left(\psi_{\infty}^{t}\right), \quad \exists\left(u_{n}\right) \subset H ; \\
u_{n} \longrightarrow u \quad \text { strongly in } H, \quad \psi_{m_{n}}^{t}\left(u_{n}\right) \longrightarrow \psi_{\infty}^{t}(u) .
\end{gathered}
$$

Our next claim is

$$
\begin{gathered}
\forall\left(u_{n}\right) \subset H \quad \text { satisfying } u_{n} \longrightarrow u \quad \text { weakly in } H, \\
\liminf _{n \rightarrow+\infty} \psi_{m_{n}}^{t}\left(u_{n}\right) \geq \psi_{\infty}^{t}(u) .
\end{gathered}
$$

For the case where $u \in D\left(\psi_{\infty}^{t}\right)=\kappa^{t}$, (3.69) follows immediately; for the case where $u \notin \kappa^{t}$, to obtain a contradiction, suppose that

$$
\exists\left(u_{n}\right) \subset H ; \quad u_{n} \longrightarrow u \quad \text { weakly in } H, \quad \liminf _{n \rightarrow+\infty} \psi_{m_{n}}^{t}\left(u_{n}\right)<\psi_{\infty}^{t}(u)=+\infty .
$$

Then we can extract a subsequence $\left(n^{\prime}\right)$ of $(n)$ such that

$$
\psi_{m_{n^{\prime}}}^{t}\left(u_{n^{\prime}}\right) \leq C \quad \forall n^{\prime}
$$

We write $m$ and $u_{m}$ simply for $m_{n^{\prime}}$ and $u_{n^{\prime}}$, respectively. Thus we have

$$
\left\{\int_{\Omega}\left(\frac{\left|u_{m}(x)\right|}{\gamma(x, t)}\right)^{m} d x\right\}^{1 / m} \leq\left\{m \psi_{m}^{t}\left(u_{m}\right)\right\}^{1 / m} \leq(m C)^{1 / m} \longrightarrow 1
$$

as $m \rightarrow+\infty$. Then in much the same way as in the proof of Lemma 3.6, we obtain

$$
\left|\frac{u}{\gamma(\cdot, t)}\right|_{L^{\infty}(\Omega)} \leq 1
$$

which contradicts the fact that $u \notin \kappa^{t}$. Therefore (3.69) follows.

Proof of Theorem 3.13. Just as in the proof of Theorem 3.5, we can derive $\left\{\psi_{m}^{t}\right\}_{t \in[0, T]} \in$ $B\left(\alpha_{2}, 0, C_{0},\left\{M_{r}\right\}_{r \geq 0}\right)$ for some constants $C_{0}>0,\left\{M_{r}\right\}_{r \geq 0}$ independent of $n$. Moreover, recall (3.57). Then for all $t_{0} \in[0, T]$ and $u_{0} \in \kappa^{t_{0}}$, we have

$$
\left\langle u(t)-u_{0}, \phi\right\rangle_{H_{0}^{1}(\Omega)} \leq\left|\gamma(\cdot, t)-\gamma\left(\cdot, t_{0}\right)\right|_{L^{\infty}(\Omega)}|\Omega|^{1 / m}|\phi|_{L^{m^{\prime}}(\Omega)},
$$

which together with (3.58) implies

$$
\left|u(t)-u_{0}\right|_{H} \leq C(|\Omega|+1)^{2}\left|\gamma(\cdot, t)-\gamma\left(\cdot, t_{0}\right)\right|_{L^{\infty}(\Omega)}
$$

Furthermore, we can easily check $\psi_{\infty}^{t}(u(t))=\psi_{\infty}^{t_{0}}\left(u_{0}\right)=0$. Hence $\left\{\psi_{\infty}^{t}\right\}_{t \in[0, T]} \in \Psi\left(\tilde{\alpha}_{2}, 0\right)$ with $\tilde{\alpha}_{2}(\cdot):=(|\Omega|+1) \alpha_{2}(\cdot)$. Therefore by Theorem 2.7 and Lemma 3.14, we complete the proof. 
Remark 3.15. In [6], the authors rewrite (3.5) in the form

$$
\frac{d u_{n}}{d t}(t)+A_{n} u_{n}(t)=0 \quad \text { in } L^{1}(\Omega)
$$

where $A_{n}$ is an $m$-accretive operator in $L^{1}(\Omega)$, and employ an abstract theory of evolution equations governed by $m$-accretive operators in a general Banach space developed in $[5$, $7,8]$. Particularly the strong convergence of $\left(u_{n}\right)$ in $C\left([0, T] ; L^{1}(\Omega)\right)$ is derived from the convergence of the resolvent of $A_{n}$, which means

$$
\left(I+A_{n}\right)^{-1} u \longrightarrow(I+A)^{-1} u \quad \text { strongly in } L^{1}(\Omega) \forall u \in L^{1}(\Omega)
$$

as $n \rightarrow+\infty$ for some $m$-accretive operator $A$. We here note that the following conditions are equivalent to each other:

(i) $\psi_{m} \rightarrow \psi_{\infty}$ on $H:=H^{-1}(\Omega)$ in the sense of Mosco as $m \rightarrow+\infty$;

(ii) $\left(I+\partial_{H} \psi_{m}\right)^{-1} u \rightarrow\left(I+\partial_{H} \psi_{\infty}\right)^{-1} u$ strongly in $H$ for all $u \in H$.

Hence our approach could also be regarded as an $H^{-1}(\Omega)$-framework version of [6].

Problem 3.16. Find a solution of the periodic problem for $\left((\mathrm{PM})_{m}\right)$ with the boundary condition $u(x, t)=0,(x, t) \in \partial \Omega \times(0, T)$, and the periodic condition $u(x, 0)=u(x, T)$, $x \in \Omega$, which is denoted by $(\mathrm{PP} 2)_{m}$. Moreover, investigate the asymptotic behavior of $u_{m}$ as $m \rightarrow+\infty$.

Just as in Problem 3.11, (PP2) $)_{m}$ is equivalent to $\left(\operatorname{PP}\left(\psi_{m}^{t}, f_{m}\right)\right)$. Concerning the existence of strong solutions, we have the following.

Theorem 3.17. Suppose that (H2) is satisfied and let $m \in[2,+\infty)$. Then for all $f \in L^{2}(0, T$; $\left.H^{-1}(\Omega)\right),\left(\mathrm{PP}\left(\psi_{m}^{t}, f\right)\right)$ has the unique strong solution $u_{m}$.

Proof of Theorem 3.17. We observe

$$
\begin{aligned}
|u|_{L^{2}(\Omega)}^{2} & \leq|\gamma|_{L^{\infty}(Q)}^{2}\left\{\frac{2}{m} \int_{\Omega}\left(\frac{|u(x)|}{\gamma(x, t)}\right)^{m} d x+\frac{m-2}{m}|\Omega|\right\} \\
& =2|\gamma|_{L^{\infty}(Q)}^{2}\left\{\psi_{m}^{t}(u)+|\Omega|\right\} \quad \forall u \in L^{m}(\Omega), \forall m \geq 2,
\end{aligned}
$$

where $Q:=\Omega \times[0, T]$. Hence since $L^{2}(\Omega)$ is continuously embedded in $H$, we can take a positive number $C_{0}$ independent of $m$ such that

$$
|u|_{H}^{2} \leq C_{0}\left(\psi_{m}^{t}(u)+1\right) \quad \forall u \in D\left(\psi_{m}^{t}\right), \forall t \in[0, T] .
$$

Therefore on account of (3.51) and (3.60), we have

$$
\left\{\psi_{m}^{t}\right\}_{t \in[0, T]} \in \Psi_{\pi}\left(\alpha_{2}, 0, C_{0}\right)
$$

Thus Theorem 2.9 ensures the existence of a strong solution $u_{m}$ of $\left(\operatorname{PP}\left(\psi_{m}^{t}, f\right)\right)$. Moreover, since $\psi_{m}^{t}$ is strictly convex on $H$, every periodic solution is unique.

As for the convergence of $u_{m}$ as $m \rightarrow+\infty$, our result is the following. 
Theorem 3.18. Suppose that (H2) is satisfied and that $\gamma(x, 0) \geq \gamma(x, T)$ for a.e. $x \in \Omega$. Let $\left(m_{n}\right)$ be a sequence in $[2,+\infty)$ such that $m_{n} \rightarrow+\infty$ as $n \rightarrow+\infty$. Moreover, let $f_{n}, f \in$ $L^{2}\left(0, T ; H^{-1}(\Omega)\right)$ be such that

$$
f_{n} \longrightarrow f \quad \text { weakly in } L^{2}\left(0, T ; H^{-1}(\Omega)\right)
$$

Then a subsequence $\left(n_{k}\right)$ of $(n)$ can be taken such that the unique strong solution $u_{n_{k}}$ of $\left(\operatorname{PP}\left(\psi_{m_{n_{k}}}^{t}, f_{n_{k}}\right)\right)$ converges to $u$ as $k \rightarrow+\infty$ in the following sense:

$$
u_{n_{k}} \longrightarrow u \text { strongly in } C\left([0, T] ; H^{-1}(\Omega)\right) \text {, weakly in } W^{1,2}\left(0, T ; H^{-1}(\Omega)\right) \text {. }
$$

Moreover, the limit $u$ is a strong solution of $\left(\mathrm{PP}\left(\psi_{\infty}^{t}, f\right)\right)$.

Proof of Theorem 3.18. We claim that any sequence $\left(u_{n}\right)$ in $H$ satisfying

$$
\sup _{n \in \mathbb{N}}\left\{\psi_{m_{n}}^{t}\left(u_{n}\right)+\left|u_{n}\right|_{H}\right\} \leq \lambda
$$

is precompact in $H$ for every $\lambda>0$ and $t \in[0, T]$. For every $m_{n} \geq 2$, we get

$$
\begin{aligned}
\left(\int_{\Omega}\left|u_{n}(x)\right|^{2} d x\right)^{1 / 2} & \leq\left(\int_{\Omega}\left|u_{n}(x)\right|^{m_{n}} d x\right)^{1 / m_{n}}|\Omega|^{\left(m_{n}-2\right) /\left(2 m_{n}\right)} \\
& \leq|\gamma(\cdot, t)|_{L^{\infty}(\Omega)}\left(m_{n} \lambda\right)^{1 / m_{n}}|\Omega|^{\left(m_{n}-2\right) /\left(2 m_{n}\right)} \leq C,
\end{aligned}
$$

where $C$ denotes a constant independent of $n$. Then since $L^{2}(\Omega)$ is compactly embedded in $H$, it follows immediately that $\left(u_{n}\right)$ becomes precompact in $H$. Moreover, since $\gamma(x, 0) \geq \gamma(x, T)$ for a.e. $x \in \Omega$, we can easily see that $\psi_{m}^{T}(u) \geq \psi_{m}^{0}(u)$ for all $u \in D\left(\psi_{m}^{T}\right)$ and $n \in \mathbb{N}$. Then the rest of proof can be derived just as in the proof of Theorem 3.9.

\section{Acknowledgments}

This is part of the author's Ph.D. thesis. The author would like to express his sincere thanks to his supervisor Professor Mitsuharu Ôtani for his constant help. Furthermore, the author is supported by Waseda University Grant for Special Research Projects, no.2003 A-123.

\section{References}

[1] G. Akagi and M. Ôtani, Time-dependent constraint problems arising from macroscopic criticalstate models for type-II superconductivity and their approximations, to appear in Adv. Math. Sci. Appl.

[2] G. Aronsson, L. C. Evans, and Y. Wu, Fast/slow diffusion and growing sandpiles, J. Differential Equations 131 (1996), no. 2, 304-335.

[3] H. Attouch, Variational Convergence for Functions and Operators, Applicable Mathematics Series, Pitman, Massachusetts, 1984.

[4] J. W. Barrett and L. Prigozhin, Bean's critical-state model as the $p \rightarrow \infty$ limit of an evolutionary p-Laplacian equation, Nonlinear Anal. Ser. A: Theory Methods 42 (2000), no. 6, 977-993.

[5] P. Bénilan, H. Brézis, and M. G. Crandall, A semilinear equation in $L^{1}\left(R^{N}\right)$, Ann. Scuola Norm. Sup. Pisa Cl. Sci. (4) 2 (1975), no. 4, 523-555. 
[6] P. Bénilan and M. G. Crandall, The continuous dependence on $\varphi$ of solutions of $u_{t}-\Delta \varphi(u)=0$, Indiana Univ. Math. J. 30 (1981), no. 2, 161-177.

[7] M. G. Crandall, An introduction to evolution governed by accretive operators, Dynamical Systems Vol. I (Proc. Internat. Sympos., Brown Univ., Providence, RI, 1974), Academic Press, New York, 1976, pp. 131-165.

[8] L. C. Evans, Application of nonlinear semigroup theory to certain partial differential equations, Nonlinear Evolution Equations (Proc. Sympos., Univ. Wisconsin, Madison, Wis, 1977), Publ. Math. Res. Center Univ. Wisconsin, vol. 40, Academic Press, New York, 1978, pp. 163 188.

[9] N. Kenmochi, Some nonlinear parabolic variational inequalities, Israel J. Math. 22 (1975), no. 34, 304-331.

[10] Solvability of nonlinear evolution equations with time-dependent constraints and applications, Bull. Fac. Education, Chiba Univ. 30 (1981), 1-87.

[11] M. Ôtani, Nonmonotone perturbations for nonlinear parabolic equations associated with subdifferential operators, periodic problems, J. Differential Equations 54 (1984), no. 2, 248-273.

[12] , Nonlinear evolution equations with time-dependent constraints, Adv. Math. Sci. Appl. 3 (1993/94), no. special issue, 383-399.

[13] H.-M. Yin, On a p-Laplacian type of evolution system and applications to the Bean model in the type-II superconductivity theory, Quart. Appl. Math. 59 (2001), no. 1, 47-66.

Goro Akagi: Department of Applied Physics, School of Science and Engineering, Waseda University, 3-4-1 Okubo Shinjuku-ku, Tokyo 169-8555, Japan

E-mail address: goro@toki.waseda.jp 


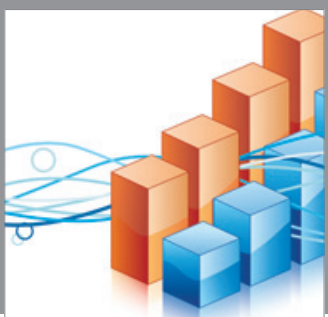

Advances in

Operations Research

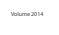

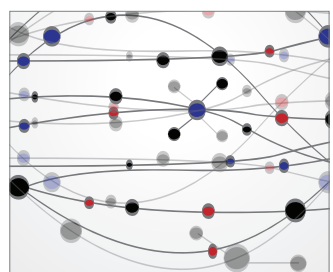

\section{The Scientific} World Journal
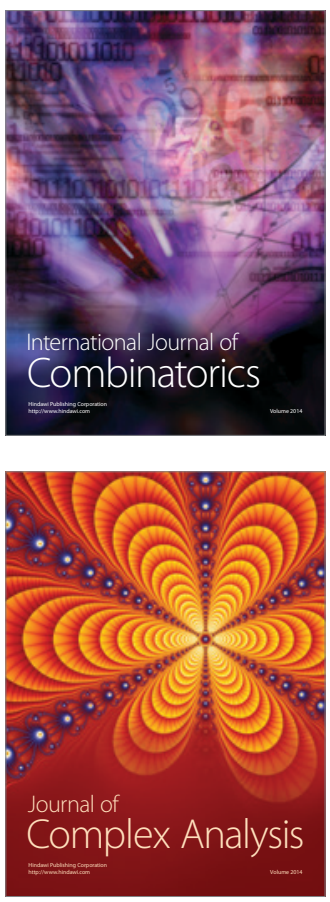

International Journal of

Mathematics and

Mathematical

Sciences
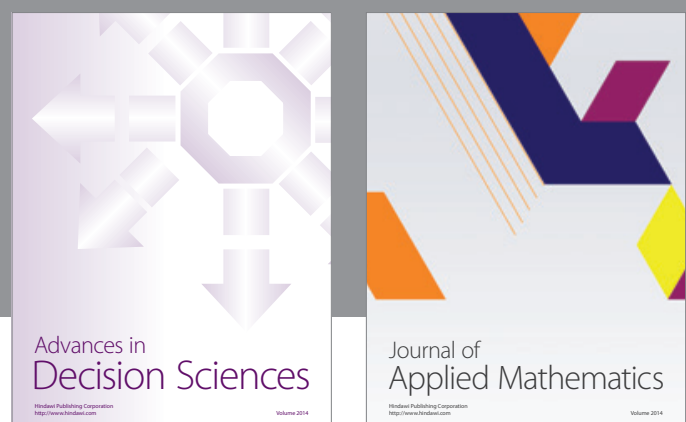

Journal of

Applied Mathematics
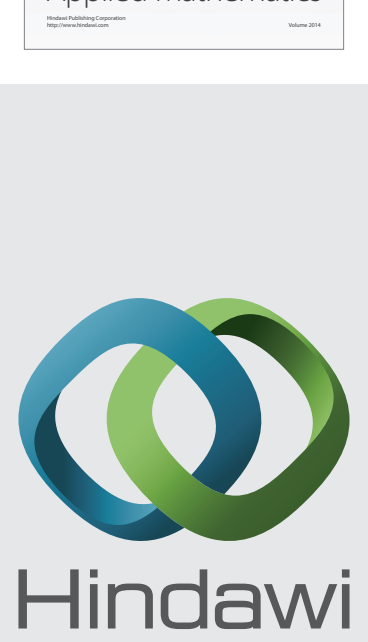

Submit your manuscripts at http://www.hindawi.com
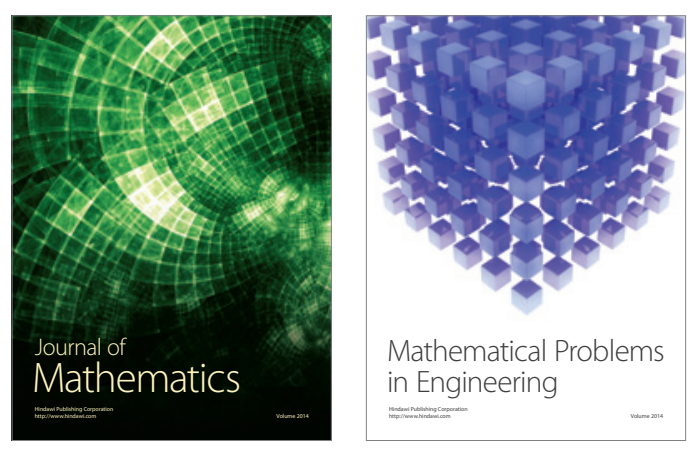

Mathematical Problems in Engineering
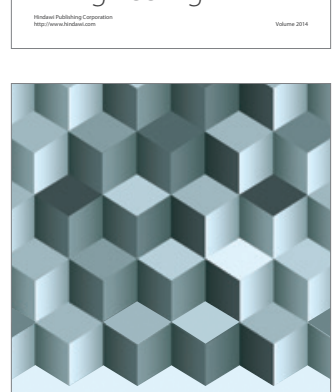

Journal of

Function Spaces
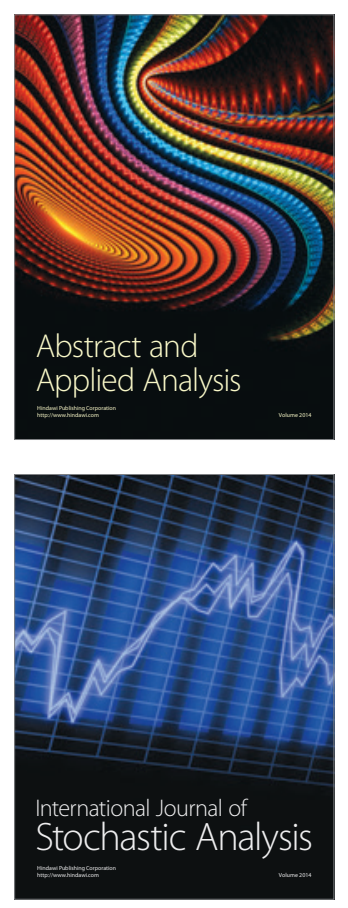

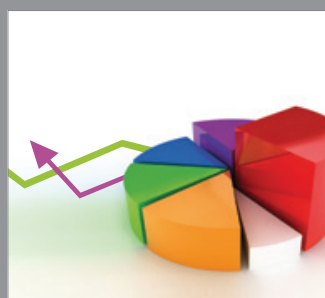

ournal of

Probability and Statistics

Promensencen
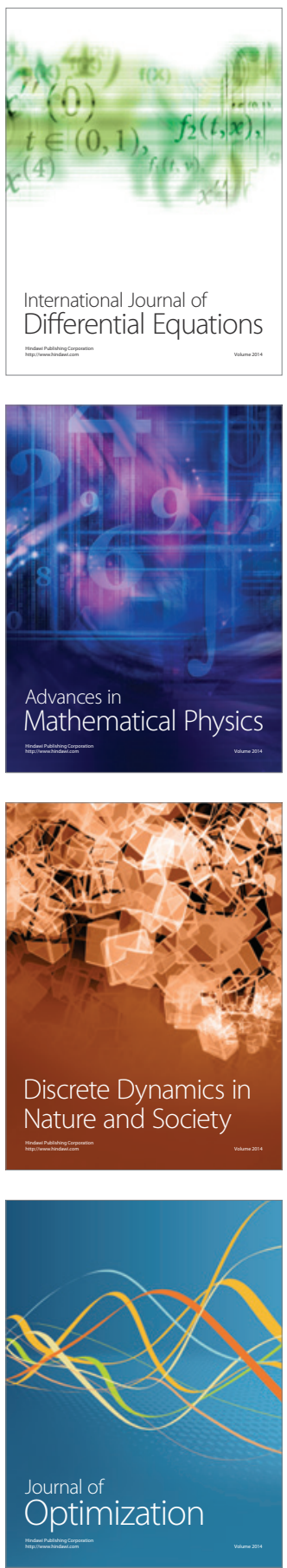